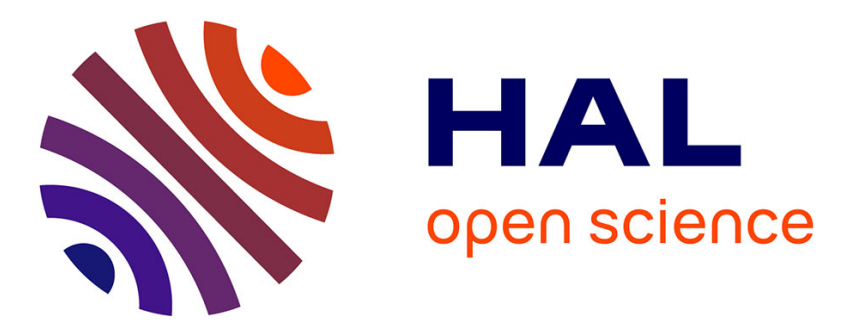

\title{
La viticulture antique en Aquitaine
}

Catherine Balmelle, Dany Barraud, Béatrice Duprat, Hervé Gaillard, Philippe

Jacques, Louis Maurin, Catherine Petit-Aupert, Didier Rigal, Karine Robin, Philippe Roudié, et al.

\section{To cite this version:}

Catherine Balmelle, Dany Barraud, Béatrice Duprat, Hervé Gaillard, Philippe Jacques, et al.. La viticulture antique en Aquitaine. Gallia - Archéologie de la France antique, 2001, La viticulture en Gaule, 58, pp.129-164. 10.3406/galia.2001.3177 . hal-01910146

\section{HAL Id: hal-01910146 \\ https://hal.science/hal-01910146}

Submitted on 20 Jan 2020

HAL is a multi-disciplinary open access archive for the deposit and dissemination of scientific research documents, whether they are published or not. The documents may come from teaching and research institutions in France or abroad, or from public or private research centers.
L'archive ouverte pluridisciplinaire HAL, est destinée au dépôt et à la diffusion de documents scientifiques de niveau recherche, publiés ou non, émanant des établissements d'enseignement et de recherche français ou étrangers, des laboratoires publics ou privés.

\section{(ㅇ)(1) $\$$}

Distributed under a Creative Commons Attribution - NonCommercial - NoDerivatives| 4.0 


\title{
LA VITICULTURE ANTIQUE EN AQUITAINE
}

\author{
Catherine Balmelle, Dany Barraud, Jean-Pierre Brun, Philippe Duprat, \\ Hervé Gaillard, Philippe Jacques, Louis Maurin, Catherine Petit-AuPert, \\ Didier Rigal, Karine Robin, Philippe Roudié, Pierre Sillières, Christian Vernou
}

\begin{abstract}
Mots-clés. Aquitaine, viticulture, Haut-Empire, Antiquité tardive, installations vinicoles, cuves de recueil du mô̂t, instruments en bois, serpe à talon-serpette, Biturica-Biturigiaca.

Key-words. Aquitaine, viticulture, Early Empire, Late Antiquity, viticultural installations, vintage tubs for the must, wooden implements, pruning-knife, Biturica-Biturigiaca.

Résumé. Le présent bilan est le fruit d'un travail d'équipe qui a été mené entre 1997 et 1999 dans le cadre d'un programme collectif de recherche sur la viticulture antique en Aquitaine, centré à l'origine sur les installations de production reconnues ou supposées dans plusieurs départements au nord et au sud de la Garonne (Charente-Maritime, Charente, Dordogne, Gironde, Lot-et-Garonne, Lot, Gers, Tarn et Haute-Garonne).

L'article s'organise en deux parties complémentaires. La première met en évidence les principales sources d'information sur la viticulture en Aquitaine : témoignages littéraires et surtout documentation archéologique fournie par les travaux sur les amphores vinaires et entièrement renouvelée par l'apport des fouilles récentes d'établissements vinicoles (Bapteste, Belmonl, Les Chapelles, Lestagnac...). Des exposés synthétiques évoquent ensuite différents aspects de la production du vin, depuis les cépages et les outils jusqu'aux techniques agricoles et à la vinification. Un essai de chronologie clôt l'ensemble de l'étude.
\end{abstract}

\begin{abstract}
The present work is the resuit of a team effort conducted from $199 \overline{9}$ to 1999 in a multidisciplinary program of research dealing with vine growing in Aquitaine. From the outset, this research was focused on known or presumed wine-making facilities in several departments located north and south of the Garonne (Charente-Maritime, Charente, Dordogne, Gironde, Lot-el-Garonne, Lol, Gers, Tarn and Haute-Garonne).

The article is divided into two complementary parts. The first treats of the chief sources of information about vine growing in Aquilaine : literary accounts and especially archaeological documentation gained from research on wine amphorae, which has been richly supplemented by recent excavations of wine-making establishments (Bapteste, Belmont, Les Chapelles, Lestagnac...). In addition, interpretative essays discuss assorted aspects of wine production, including varieties of wine and tools, as well as techniques for cultivating and processing wine. A chronological study completes the work.
\end{abstract}

(traduction D. Parrish).

Le profond renouvellement des connaissances sur les installations de production de vin en Méditerranée à l'époque antique au cours de ces dernières années ${ }^{156} \mathrm{a}$ incité un petit groupe de chercheurs à examiner d'un œil nouveau le dossier de la viticulture en Aquitaine gallo-romaine et à entreprendre en particulier une enquête approfondie sur les bâtiments dotés de bassins à

156. Amouretti, Brun (éds), 1993 ; Comas (ed.), 1998. cuvette de vidange qui ont été découverts dans le SudOuest ${ }^{157}$. Le panorama que nous présentons, fruit de ce travail d'équipe mené entre 1997 et 1999, est organisé en

157. À l'initiative de C. Balmelle (CNRS-ENS, Paris) qui avait été amenée à réfléchir sur la viticulture en Aquitaine à la fin de l'Antiquité, en rédigeant un ouvrage sur les demeures aristocratiques de ces régions de la Gaule (Balmelle, 2001), un programme collectif de recherche a été mis en place en 1997 avec D. Barraud (SRA, Aquitaine), J.-P. Brun (CNRS, Centre Camille-Jullian), L. Maurin (Ausonius, Universitć 
deux volets. Le premier expose la documentation archéologique essentielle livrée par une dizaine de sites, sous forme de courtes notices illustrées. Le second regroupe une série d'exposés synthétiques qui traitent de différents aspects de la production du vin, depuis les cépages et les outils, jusqu'aux techniques agricoles et à la vinification. Un essai de chronologie clôt l'ensemble de cette étude.

Le thème de recherche n'est pas nouveau : les sources textuelles comme divers travaux archéologiques ont déjà attiré l'attention sur la viticulture dans le sud-ouest de la Gaule.

Columelle et Pline l'Ancien, dans la seconde moitié du I ${ }^{\mathrm{cr}} \mathrm{s}$. après J.-C., connaissent un cépage d'une certaine notoriété, nommé Biturica par le premier, Biturigiaca par le second.

Les références les plus anciennes et les plus nombreuses sont fournies par le livre III du traité d'agriculture de Columelle (III, 2, 19-20, p. $13 ; 2,28$, p. $17 ; 7$, 1 , p. $31 ; 9,1$, p. $35 ; 9,3$, p. $36 ; 9,7)$. Au début du livre, après la description des cépages de crus précieux, l'écrivain ajoute :

"[...] Possunt tamen etiam secundae notae uiles prouentu et ubertate commendari, qualis Biturica, qualis balisca, quarum minorem coccolobin uocant Hispani, longe omnium primis utraeque proximae."

"On peut pourtant recommander des vignes de seconde qualité, qui poussent et rendent bien, comme la Biturique, comme la balisca dont les Espagnols appellent la plus petite sorte coccolobis: elles sont toutes deux de bien loin celles qui s'approchent le plus du premier rang. " (Columelle, De l'agricullure, III, 2, 19, trad. J.-C. Dumont, p. 13).

Columelle précise dans un autre passage que la Biturica a été réccmment importée de pays lointains (III, 9, 3, p. 35-36).

Quelques années plus tard, Pline l'Ancien, dans sa célèbre énumération des plants italiens, gaulois et

Bordeaux 3), C. Petit-Aupert, P. Roudié (Université Bordeaux 3), P. Sillic̀res (Ausonius, CNRS), C. Vernou (Service du patrimoine, Conseil général de la (harente). L'équipe s'est peu à peu étofféc et ent ichie avec H. Gaillard, auteur de l'ensemble des dessins, C. Goyaud, viticultrice à Barsac, P. Jacques (Association des archéologues du Lot-et('aromne) et K. Robin (AFAN), qui ont l'un et l'autre fouillé tout récemment des installations viticoles d'un intérêt particulier. P. Duprat a eu l'obligeance de nous livrer les dernières informations recueillies en 1999 sur le site de la villa de Port-des-Barques (Charente-Maritime). espagnols, mentionne la Biturigiaca, de façon assez brève : elle n'a pas de renom particulier, mais on en tire un vin qui vieillit bien ( $H . N$., XIV, 27, p. 32).

À la fin de l'Antiquité, on retrouve la mention du plant Biturica sous la plume d'Isidore de Séville (Élymologies, XVII, 5, 22, p. 58-59) ; l'écrivain semble s'inspirer d'un passage de Columelle (III, 2, 20, p. 13).

Bien qu'aucun de ces textes ne permette d'identifier avec certitude la région où était cultivée la Vitis Biturica, on peut supposer avec une certaine vraisemblance, à la suite de C. Jullian ${ }^{158}$, R. Dion ${ }^{159}$, R. Étienne ${ }^{160}$, que ce terme latin désigne un cépage du pays des Bituriges Vivisques (plutôt que des Bituriges Cubi). Divers indices archéologiques attestent en effet l'origine ancienne de la viticulture dans le Bordelais (voir infra, p. 150-151 et 163) ; d'autre part, les sources de l'Antiquité tardive montrent bien l'enracinement de cette culture autour de la capitale de la cité des Bituriges Vivisques.

Grâce aux textes, on pressent également l'importance et le prestige de la viticulture dans maintes campagnes du Sud-Ouest entre le $\mathrm{IV}^{\mathrm{c}}$ et le $\mathrm{VI}^{\mathrm{e}} \mathrm{s}$. Le panégyriste de Constantin, en 312, souligne qu'on avait l'habitude dans les Aquitaines - de même que dans d'autres provinces - " d'assigner n'importe où un terrain à de nouvelles vignes " (Panégyriques latins, VIII, 68, t. II, p. 95). Dans la seconde moitié du IVes., Ausone insiste sur la présence de la vigne dans les campagnes autour de Bordeaux ${ }^{161}$ et il nous apprend que le «petit » domaine qui lui vient de son père, dans la région de Bazas (Gironde), ne comporte pas moins de cent jugères de vigne, soit environ 25 ha ${ }^{162}$. $\mathrm{Au}$ siècle suivant (vers 440), Salvien, à propos des Aquitaines et de la Novempopulanie, évoque un pays couvert de vignes (De gubernatione Dei, VII, 8, p. 435-436). $\mathrm{Au} \mathrm{VI}^{\mathrm{c}}$ s. enfin, Venance Fortunat, décrivant le domaine de Léonce II à Praemiacum près de Bordeaux, mentionne " la vigne luxuriante [qui] ombrage la terre nour-

158. Jullian, rééd. 1993, vol. 2, p. 584, note 50.

159. Dion (1959, p. 412-416) suppose que la Biturica avait été importée des zones cantabriques.

160. Étiennc (1962, p. 102-107) refuse la théorie de R. Dion sur l'origine cantabrique de la Biturica et propose d'y voir un plant dont l'origine serait à rechercher en Épire.

161. En particulier Ausonc, Mosella, 160, p. 178: "sic mea flauentem pingunt uineta Garumnam ; Epist. 23 à Paulin : « uitiferi colles", p. 277.

162. Ausone, De herediolo, XII, 21-22, p. 90 : « uinea centum jugeribus». 
ricière $" 163$. Les textes permettent également d'entrevoir la place que tient la vigne dans les préoccupations des grands propriétaires, laïcs et religieux. L'un des plus évocateurs est sans doute celui de Paulin de Pella, membre de l'aristocratie sénatoriale, qui, autour de 400 , consacre ses soins aux vignobles épuisés de la propriété de sa femme, en les régénérant par un procédé nouveau ${ }^{164}$. À cette époque, Paulin vit en Aquitaine Seconde où il possède également, à Bordeaux, une domus qui lui vient de ses ancêtres.

Au regard de ces divers témoignages littéraires, les indices archéologiques d'une activité viticole/vinicole étaient, jusqu'à ces dernières années, bien maigres et ils consistaient principalement en vestiges d'installations de production, souvent mal datés et d'interprétation contestée.

Il faut rappeler qu'autour de 1957 plusieurs trouvailles de bassins agricoles à cuvette de vidange furent signalées en Gironde, à Cadillac, à Mérignas et à Loubens. Dès cette époque, J. Coupry avait émis l'hypothèse que ces installations servaient à la vinification et qu'il en allait sans doute de même pour les bassins à cuvette découverts en 1936 à Montcaret (Dordogne) (Coupry, 1959b, p. 53). Quelques années plus tard, en 1964, L. Maurin avait aussi proposé d'identifier un ensemble de constructions qu'il avait fouillé en 1957 à Allas-les-Mines (Jordogne) comme une instailation vinicole (Maurin, 1964). Depuis, d'autres bassins furent découverts, notamment dans l'Entre-Deux-Mers (Sireix, Berthault, 1990). Mais un doute subsistait sur la destination de ces bassins, en général très partiellement explorés.

De même, plus au nord de l'Aquitaine, dans les deux départements de la Charente et de la Charente-Maritime, de très nombreuses installations à batterie de bassins mises au jour depuis le XIX ${ }^{\mathrm{e}} \mathrm{s}$. avaient attiré l'attention des chercheurs (Maurin, 1978); mais là encore la question de leur destination restait problématique. Or en 1987, la fouille menée par C. Vernou d'un vaste ensemble au lieu-dit La Haute-Sarrazine à Cognac (Charente) fut l'occasion de réouvrir le dossier ; dans le

163. Venance Fortunat, Carmen, I, 20, 16, t. I, p. 45 : « pinguis et altricem palmes opacat humum".

164. Paulin de Pella, Eucharisticos, 194-198, p. 70 ; sur ce personnage qui est identifié unanimement à un petit-fils d'Ausone, voir Stroheker, 1948, n 292 et PLRE, I, s. v. Paulinus, 10, p. 677-678. catalogue d'exposition, C. Vernou a présenté toutes les hypothèses envisageables à propos de l'utilisation de ces bassins, y compris l'hypothèse vinicole, sans trancher sur la destination de l'établissement de La Haute-Sarrazine (Vernou et al., 1990).

Au cours de ces dix dernières années, un autre indice de la production du vin a été décelé dans la production d'amphores vinaires. Ainsi les travaux menés par F. Berthault laissent aujourd'hui supposer le développement au $\mathrm{I}^{\mathrm{er}} \mathbf{s}$. de plusieurs productions locales d'amphores à Bordeaux, à Agen, à Saintes et à Périgueux (Berthault, 1998). Les ateliers sont toutefois encore mal connus, hormis celui de Saintes qui a produit des imitations d'amphores Dressel 2/4 et Gauloise 3/5 (Michaud, 1991 ; Berthault, 1991). Dans les régions plus méridionales, T. Martin a récemment identifié une production d'amphores vinaires à Montans (Tarn), qui remonte également au I ${ }^{\mathrm{er}} \mathrm{s}$. (Martin, 1996, p. 44-45). Sans doute n'est-il pas inutile de souligner dès à présent que la totalité de ces témoignages concerne exclusivement les débuts de la viticulture aquitaine et, qu'en l'état actuel des connaissances, le matériel amphorique n'apporte aucune donnée précise sur la production du vin à partir du milieu du II $^{\mathrm{e}} \mathrm{s}$.

Le programme collectif de recherche triennal qui a été lancé en 1997 sur la viticulture dans l'Aquitaine galloromaine a eu pour principal objectif de recenser tous les vestiges susceptibles d'être identifiés comme des instruments ou des installations liés à la culture de la vigne et à la production de vin, depuis les serpettes de vendangeurs jusqu'aux fouloirs et aux cuves. Ont été aussi pris en compte des vestiges d'ordre différent : traces de culture, pépins et sarments, tonneaux, dolia ${ }^{165}$.

En ce qui concerne les installations de production, deux principaux critères distinctifs ont été retenus pour la sélection des sites: la présence d'une cuvette de vidange au fond du bassin et la disposition en batterie des structures. Dans une seconde étape, on a opéré, lors de réunions de concertation, une double sélection sur l'ensemble des sites recensés en vue de définir les établissements qui sont liés de façon sûre ou probable à la production de vin et ceux sur lesquels des recherches archéologiques pourraient être entreprises ultérieure-

165. Pour les amphores vinaires, nous nous sommes appuyés sur l'ensemble des travaux récemment publiés sur le sujet, en particulier ceux de F. Berthault. 
ment. Il faut remarquer que contrairement à ce que l'on avait supposé antérieurement, aucun site à batterie de bassins rencontré en Charente-Maritime sur le littoral ne semble correspondre de façon assurée à des établissements de salaison. L'organisation générale des bassins en particulier n'est nullement comparable à celle des fabriques de salaisons de poisson.

La base documentaire réalisée dans ce programme a couvert neuf départements qui relèvent de trois régions distinctes: Charente et Charente-Maritime en PoitouCharentes, Dordogne, Girondc, Lot-et-Garonne en Aquitaine, Gers, Haute-Garonne, Lot, Tarn en MidiPyrénées. À côté de cette base qui comporte actuellement plus de cent dix fiches ${ }^{166}$, une recherche plus approfondie sur les outils de vignerons a été menée par Christian et Claudine Vernou.

C. B. et L. M.

\section{LES INSTALLATIONS VINICOLES}

\section{LA GÉOGRAPHIE DES VIGNOBLES}

La carte publiée ici (fig. 61) regroupe les principaux indices d'une culture de la vigne répertoriés dans les départements que nous venons d'énumérer. Il a paru souhaitable, pour des questions de clarté, de dissocier les trouvailles de serpes et de serpettes qui sont détaillées sur une autre carte accompagnant la présentation sur les outils de vignerons (fig. 77).

À petite échelle, la répartition des vestiges d'une activité viticole dans l'Aquitaine antique affirme d'emblée l'importance de deux des vignobles aujourd'hui parmi les plus vastes et les plus réputés du monde, à savoir les pays charentais de Cognac et le Bordelais, au détriment de secteurs périphériques en principe défavorables à la production viticole, tels les Landes et le Limousin.

Mais cette constatation a priori rassurante pour l'argumentaire d'une grande ancienneté des deux vignobles précités doit être complétée par des analyses plus précises. Car on ne peut qu'être surpris de la grande

166. La répartition très inégale selon les départements est la suivante: 43 en Charente-Maritime, 31 en Charente, 22 en Gironde, 6 en Dordogne, 4 dans le Lotet-Garonne, 2 dans le Lot, 3 dans le Gers, 2 dans le Tarn, 1 en Haute-Garonne. rareté des vestiges dans nombre de campagnes du SudOuest qui ont porté et possèdent encore aujourd'hui de beaux vignobles : le Périgord, le Haut-Quercy, l'Agenais, le Toulousain, y compris dans les grandes vallées (Lot, Tarn, Dordogne, Garonne même), ne révèlent en effet que des trouvailles isolées.

À l'intérieur même des deux grands vignobles aquitains antiques, Charentes et Bordelais, révélés par la carte, les contrastes sont très nets, laissant même quelques vides suspects. Dans les Charentes par exemple, deux secteurs s'affirment nettement; le premier autour de Cognac se rattache par la vallée de la Charente au second constitué par la zone littorale de l'Aunis (y compris l'île d'Oléron), en bordure de la mer des Pertuis. Le système fluvial de la Charente est même aussi bien représenté en amont de Cognac. Mais, au nord, les abords du Poitou sont vides, dans l'état actuel de la recherche, tout comme au sud-ouest de la Seudre. En Bordelais, où la densité des trouvailles est notable en Entre-Deux-Mers et en Libournais, prolongé même par le Bergeracois, l'absence de trouvaille viticole est remarquable en Médoc (à l'exception de Vertheuil) et plus encore au pays des côtes de la rive droite de l'estuaire de la Gironde en Blayais et en Bourgeais ${ }^{167}$.

De façon plus précise encore, la carte fait apparaître des dominantes : si les trouvailles d'éléments organiques de la vigne (à peine une dizaine) sont très éparpillées, tout comme celles des serpes et serpettes (fig. 77), et les très rares vestiges de pressoirs plutôt méridionaux, les découvertes des bassins et surtout celles des bassins multiples affirment la toute puissance de la Charente littorale, puis de la Saintonge de Cognac et du Bordelais. Dans le cas des Charentes, on sait qu'aujourd'hui le centre le plus dense et le plus réputé de la viticulture est au contraire celui de la "Grande Champagne » de Cognac, les zones littorales et périphériques n'ayant droil qu'aux appellations de "Bois ". Les corrélations entre les deux grands vignobles antiques révélées par la carte et la géographie ne sont pas évidentes, à ceci près que le rôle des rivières et du littoral semble important et que la présence des terres calcaires peut être invoquée comme un facteur favorable à la viticulture. Tout semble renforcer l'idée que le vignoble

167. On sait qu'à l'époque médiévale, les Bordelais ont tout fait pour empêcher l'émergence de vignobles d'aval dont la production commercialisée par la mer aurait pu leur échapper. 


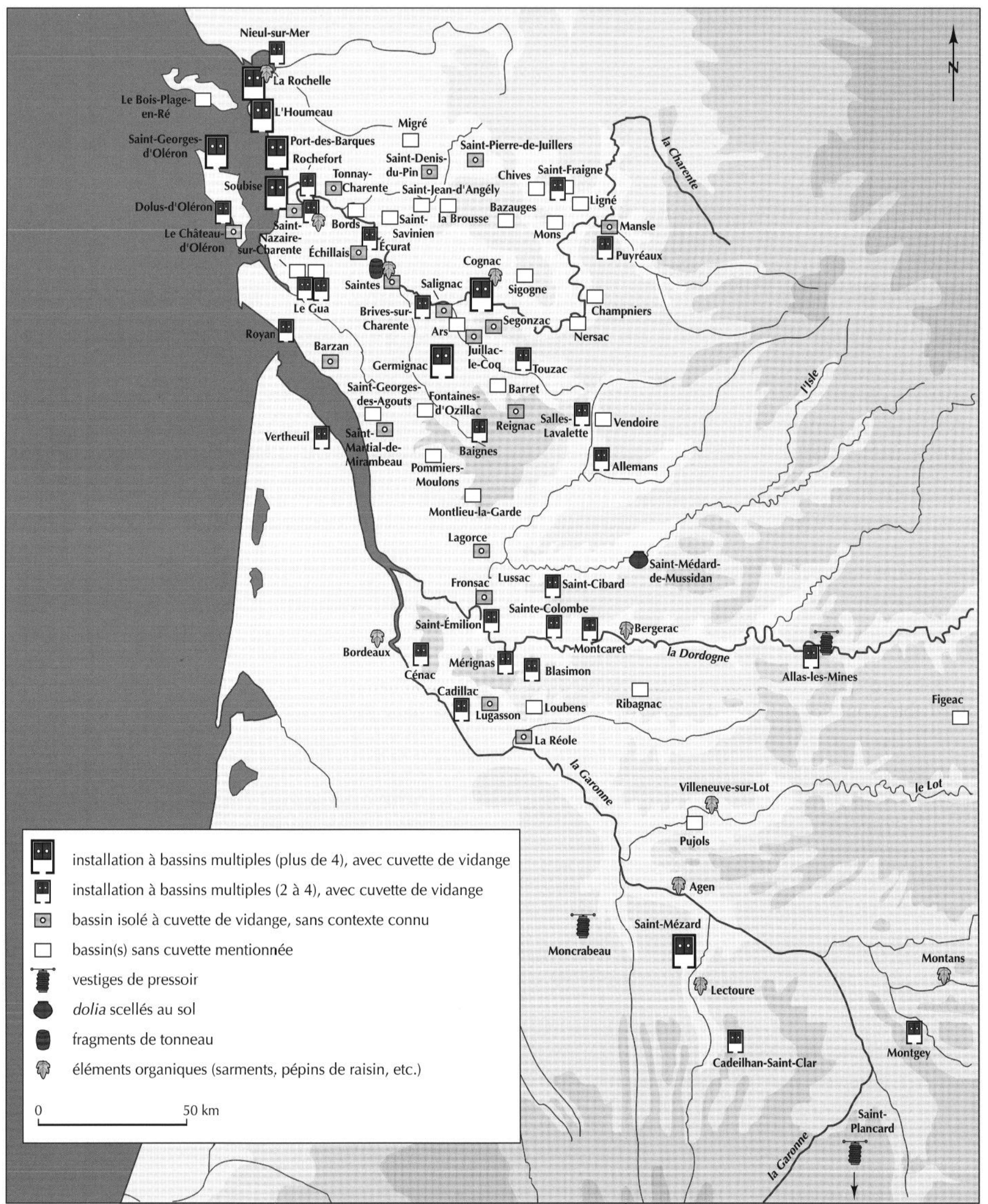

Fig. 61 - Carte des vestiges d'une activité vinicole dans l'Aquitaine gallo-romaine, d'après les travaux menés dans le cadre du programme collectif de recherche (dessin H. Gaillard, 1999). Pour les serpes et les serpettes, voir carte détaillée fig. 77. 
antique est lié à la mer et à l'eau, et que les voies de communication et les transports par bateau ont eu un rôle prééminent ${ }^{168}$.

Quant à relier la viticulture antique à celle d'aujourd'hui, il semble que ce soit un essai voué à l'échec. Trop d'épisodes importants ont bouleversé la géographie des vignobles : ainsi, de 1154 à 1453, l'Aquitaine, possession personnelle des rois d'Angleterre, fut le terroir du vignoble à vin dont avaient besoin les îles Britanniques; ou encore, à l'époque moderne, le développement de la distillation par les Hollandais, notamment dans les Charentes; ou enfin, au XIX ${ }^{\mathrm{e}}$ s., la crise du phylloxera.

On est bien obligé, enfin, d'évoquer à titre d'explication la très probable inégalité des recherches et par conséquent des trouvailles, la Charente-Maritime et le Bordelais ayant été sûrement beaucoup plus prospectés que les autres secteurs de la grande Aquitaine. Sans compter les trouvailles fortuites, les recherches en cours vont encore à coup sûr enrichir la carte. La preuve nous en est fournie par les découvertes faites récemment en Gascogne gersoise, dans le Lectourois. Les prospections aériennes de Catherine Petit-Aupert avaient permis de repérer une série de bâtiments agricoles, de plan rectangulaire allongé, dans des villae. La fouille de l'un d'entre eux, à Lestagnac, près de Saint-Mézard, en juillet 1999, a montré qu'il s'agissait de chais (voir p. 144-146) : on peut ainsi présumer que la carte des vestiges vinicoles va s'enrichir d'une dizaine de points dans ce secteur de l'Aquitaine méridionale.

P. R.

\section{QUELQUES SITES REPRÉSENTATIFS}

Les neuf notices qui suivent concernent, pour six d'entre elles, des opérations archéologiques menées entre 1987 et 1999. Les trois autres se rapportent à des fouilles effectuées antérieurement, à diverses époques. Les rédacteurs de ces dernières fiches, qui ont été dans plusieurs cas les responsables des fouilles, ont été parfois amenés à apporter des révisions aux textes déjà publiés sur ces établissements.

168. Les effets du prix des transports sur la localisation des vignobles à l'époque romaine ont été examinés par P. Sillières (1991, p. 749-757).

\section{ALLAS-LeS-Mines (DORDOGNE)}

Sur la rive gauche de la Dordogne, en un point de passage de la rivière, l'établissement antique est au contact entre la plaine alluviale qui s'élargit ici à la faveur d'un méandre et les dernières pentes des collines molles qui dominent la rivière. La partie orientale du site est implantée sur le terrain en pente douce.

À la fin de 1956 et au début de 1957, le site a été dégagé en quelques semaines pendant lesquelles des observations archéologiques et un plan sommaire ont été réalisés (Coupry, 1957, p. 240-241 et 1959a, p. 391-395 ; Maurin, 1964 ; Gaillard, 1997, p. 63-64). Aucune analyse de la stratigraphie n'a donc été faite, ce qui aurait été utile dans la partie orientale ; le matériel conservé se limitait à quelques tessons de céramique commune, à un bronze de Vespasien, un autre de Trajan et cinq antoniniens (Gallien, Postume, Claude II, Quintille, Tetricus père). L'étude a donc porté seulement sur l'architecture. Du jeu de photographies prises à l'époque, une seule a été retrouvée dans les archives du Service régional d'Aquitaine (fig. 63).

Les fouilles ont mis au jour un bâtiment cohérent et sans doute à peu près complet (fig. 62). Vers le nordouest, un fragment de mur (d sur le plan), prenant appui sur le mur de bordure, ne parait avoir aucun lien de fonctionnement avec l'ensemble. Les destructions antérieures à la fouille ont empêché de voir comment l'établissement était clos du côté oriental, où devait se trouver l'entrée. On reconnaît de l'ouest à l'est :

- Une grande salle rectangulaire $1(10,08 \mathrm{~m} \times 5,32 \mathrm{~m})$, aux murs et au sol recouverts de mortier de tuileau, avec des joints d'étanchéité au contact du sol, ce dernier en pente vers l'est. L'accès est incertain (par la salle 2 ?).

- Une construction $(10,15 \mathrm{~m} \times 6,65 \mathrm{~m})$ dont n'ont été retrouvées que les fondations des murs : quatre petites pièces symétriques (3-6) s'ordonnent autour d'une salle centrale 2. Cependant, sur une photographie prise au cours de destructions qui ont précédé l'intervention archéologique, un reste de sol de béton de tuileau dessine une flexure soutenue par la maçonnerie séparant les salles 3 et $4^{169}$; on a interprété alors cet ensemble comme deux salles allongées, comprenant en leur milieu

169. Maurin, 1964, p. 212 et fig. 6 . Cette précision (la flexure) ne peut être réfutée, car je la tiens de l'auteur de la photographie (un peu floue, il est vrai), J. Coupry, alors directeur des Antiquités historiques de Bordeaux. 


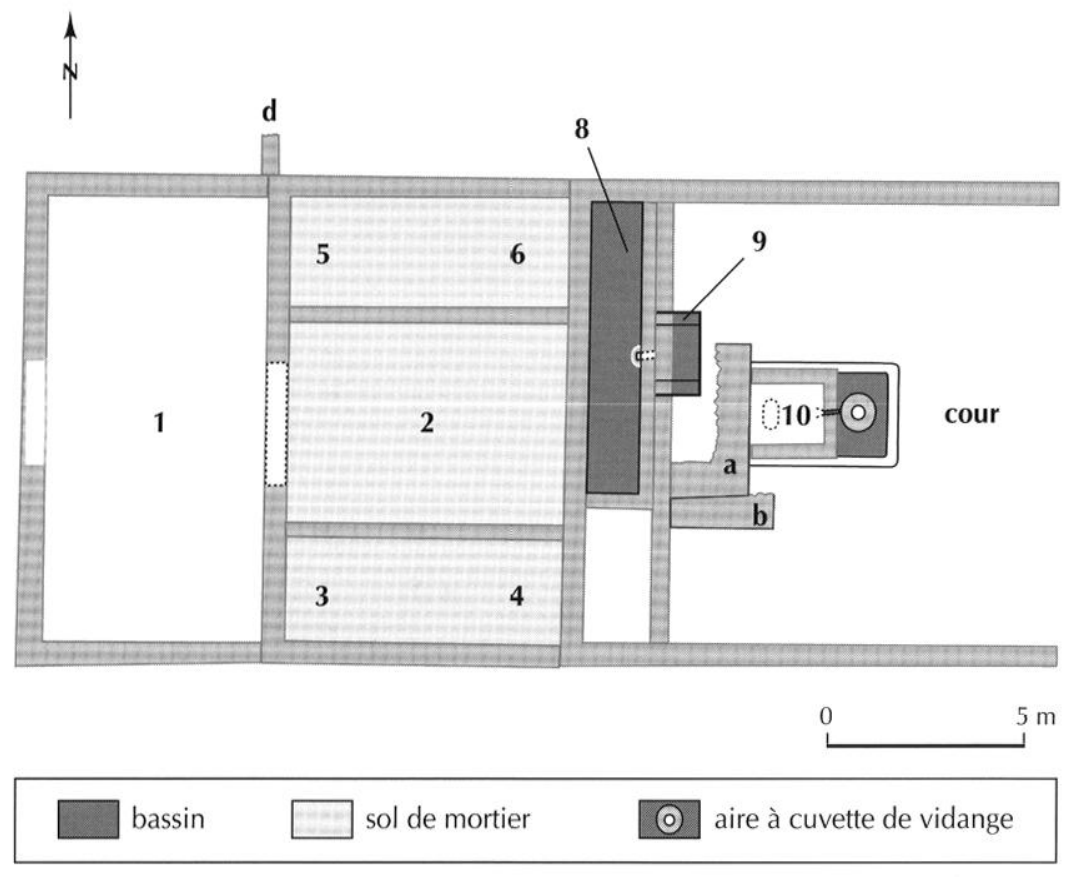

Fig. 62 - Plan de l'installation vinicole d'Allas-les-Mines, Dordogne (dessin H. Gaillard, 1999, d'après Maurin, 1964).

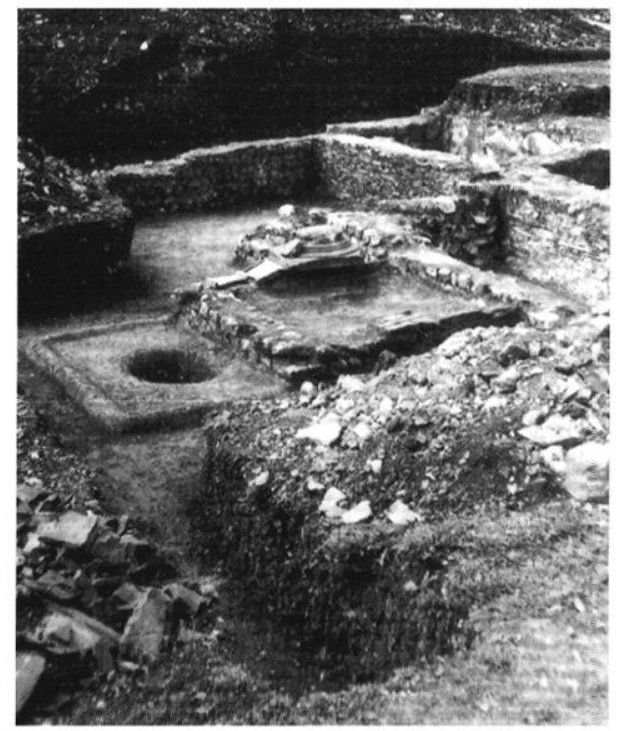

Fig. 63 - Photographie vers le sud au moment de la découverle de l'installation vinicole d'Allas-les-Mines, Dordogne : soubassement du pressoir (10) et petite curve accolée à l'est (cliché. Coupry). cette flexure, de part et d'autre de la salle 2. Ce cliché indique au moins que ces pièces, et sans doute la salle 2 , étaient, comme la salle 1, pavées d'un sol de mortier au tuileau avec pente vers l'est. L'accès est incertain : peutêtre se trouvait-il au nord.

Les salles 1-6 paraissent constituer un ensemble homogène, dont les parallèles les plus proches (au moins pour les pièces 1 et 3-6) nous semblent les aires bétonnées de l'établissement de La Haute-Sarrazine à Cognac. Elles étaient fermées à l'est, sans communication avec une vaste aire de terre battue située de ce côté en contrebas et fermée par un mur dont la plus grande élévation conservée atteignait $0,95 \mathrm{~m}$.

- Un ensemble de structures en contrebas, regroupant des constructions complexes. Au fond (à l'ouest), où les murs avaient été primitivement revêtus d'un mortier jaune, fut aménagée à une date imprécise une pièce 8 très allongée $(6,64 \mathrm{~m} \times 1,22 \mathrm{~m})$, étanche, qui s'interprète avec une quasi-certitude comme un grand fouloir; les pentes du sol aboutissent au centre de la paroi orientale à un orifice qui débouche au-dessus d'un bassin 9, lui aussi étanche, qui devait être vidé au fur et à mesure de son remplissage.

Ultérieurement, cette partie de l'établissement a été condamnée par la construction d'un mur qui, au passage, s'enfonçait jusqu'au fond du bassin 9. Contre ce mur viennent s'appuyer deux épaisses maçonneries (a et b). Jointives, elles sont parementées sur les côtés sud et est. Mais, à l'ouest, le bassin 9 et ses abords ont été, lors de l'intervention archéologique, vidés de tous les matériaux de comblement libres, avant toute observation; de même, on ne sait pas comment s'arrêtait au nord la maçonnerie $b^{170}$. Il est probable que a formait une estrade massive dont un large gradin $b$ facilitait l'accès. La condamnation des pièces 8 et 9 , qui constituaient le fouloir, avec son bassin d'évacuation, semble avoir eu pour objet de le remplacer par un pressoir $10^{171}$ (fig. 63). Appuyée au massif a, la base de celui-ci était cernée par trois murettes couvertes de tuiles

170. Sur un croquis dressé en 1956 par H. Allègre, instituteur à Allas, le mur b s'arrête au nord contre la murette du pressoir (Coupry, 1957, p. 241, fig. 1).

171. Cette interprétation diffère de celle qui a été présentée dans Gallia 1964 où l'on supposait que, dans une première étape, le fouloir et le pressoir avaient fonctionné en même temps. De même, J.-P. Brun avertit que la comparaison avec la scène de pressurage donnée par une mosaïque de Saint-Romain-en-Gal (Lancha, 1981, $\mathrm{n}^{\circ} 368$, pl. CXXI, a) ne s'imposait pas, l'artisan ayant figuré sur ce pavement un cadre architectural purement conventionnel. Il ne faut donc pas mettre en rapport le pressoir avec le chapiteau toscan découvert à proximité immédiate. 
à rebords. Elles limitaient l'aire où avait lieu le pressurage, qui devait être opéré par un prelum à l'intérieur d'une cage de bois dressée sur cette aire. Engagé dans la maçonnerie a, le prelum, grande poutre de bois, devait être abaissé à l'autre extrémité par un treuil fixé à un contrepoids massif dont on n'a pas retrouvé la trace : il pouvait se trouver en dehors de la partie conservée de l'établissement, dont tout vestige avait disparu à $3 \mathrm{~m}$ environ en avant (à l'est) du pressoir ; devant ce dernier s'ouvre un cuvon profond de $45 \mathrm{~cm}$, ménagé dans une aire de béton de tuileau; le liquide s'y écoulait par un tuyau de plomb, et il était vidé au fur et à mesure de son remplissage.

Il est hors de doute que l'on soit dans les bâtiments d'exploitation d'un établissement agricole dépendant d'une villa. Un habitat avec sols mosaiqués a été signalé aux Gourgues, au nord du village, à $300 \mathrm{~m}$ environ (Gaillard, 1997, p. 64-65). Mais le rapport avec l'établissement vinicole est évidemment hypothétique.

L'ensemble du mobilier découvert semble indiquer que l'installation vinicole a fonctionné au cours du HautEmpire, probablement jusque dans le troisième quart du III ${ }^{e}$ s., si l'on se fie au témoignage des dernières monnaies recueillies sur le site.

L. M.

\section{BAPTESTE À MONCRABEAU (LOT-ET-GARONNE)}

Le site, qui se trouve à la limite nord de la commune de Moncrabeau, occupe le bord d'une terrasse secondaire surplombant de $5 \mathrm{~m}$ la vallée de la Baïse. Les parcelles contenant la villa antique de Bapteste, fouillées entre 1871 et 1873 par Anatole Faugère-Dubourg (Faugère-Dubourg, 1875 ; Marchi, 1994 ${ }^{172}$ ), ont été rachetées en 1992 par la municipalité de Moncrabeau pour mettre en valeur les vestiges mis au jour au XIX ${ }^{e} s$. Les sondages réalisés en 1992 ont révélé des structures très dégradées (Rouzeau, 1995). Vu ces résultats, les dernières campagnes de fouille qui ont été menées ont consisté à définir l'évolution générale du site (Jacques, 1996).

L'occupation primitive correspond à un petit habitat datable de l'âge du Bronze. La première villa, du type à

172. Soulignons qu'il s'agit d'un mémoire secondaire d'un diplôme d'études supérieures (DES), soutenu à Bordeaux en 1965 sous la direction de J. Coupry. Le texte a été publié tel quel en 1994. galerie de façade avec ailes en retour d'angle, semble avoir été implantée dans la seconde moitié du $\mathrm{I}^{\text {er }} \mathrm{s}$. (état 1). Par la suite, ce bâtiment connaît des transformations successives, notamment au $\mathrm{II}^{\mathrm{e}} \mathrm{s}$. où l'on aménage un pressoir puis une cuisine (états 2 et 3 ). Cette villa fut rasée dans le deuxième quart du $\mathrm{IV}^{\mathrm{e}} \mathrm{s}$. pour laisser place à un nouvel édifice centré sur une cour carrée à péristyle (état 4). Cette seconde villa connut son expansion maximale à la fin du IV $V^{\mathrm{e}}$ ou au début du $\mathrm{V}^{\mathrm{e}} \mathrm{s}$. après J.-C. (création d'une cour d'honneur et d'une annexe thermale).

\section{Les installations agricoles de l'état $\mathbf{2}$ de la villa}

Dans cette phase, deux murs M11 et M18, dont l'angle est formé par un bloc monolithe en calcaire, furent ajoutés à l'édifice primitif (fig. 64). Le mur M11 présente sur sa face extérieure deux arases de briques régulièrement séparées par trois assises de moellons.

Le sol en terre de la pièce $\mathrm{D}$ présente des empreintes. L'une d'elles, rectiligne, séparant l'espace en deux, pourrait être interprétée comme la trace laissée par une cloison en bois. Deux observations confortent cette hypothèse : de part et d'autre de cette limite, le niveau des sols présente une différence de 2 à $3 \mathrm{~cm}$; de plus, quatre empreintes de planches juxtaposées ( $\mathrm{St}$ 2) semblent correspondre aux vestiges de la cloison effondrée. À l'ouest de cette dernière se trouve une fosse creusée dans l'argile naturelle (St1). De forme tronconique à fond convexe, elle marque probablement l'emplacement d'un dolium. À l'est de la cloison, le sol montre une empreinte $(\mathrm{St} 4)$ en forme de $« \mathrm{H} »(2,50 \mathrm{~m} \times$ $1,50 \mathrm{~m}$ ), trace d'un socle en bois (fig. 65). La forme permet de comprendre comment ce socle était assemblé : une traverse centrale s'emboîtait dans deux pièces de bois latérales faisant office de pieds. Ce support pourrait être la base d'un pressoir à vis directe en bois. La structure en creux est entourée par une dépression de forme grossièrement circulaire qui serait la trace laissée par le piétinement des servants du pressoir. L'association pressoir et dolium est souvent rencontrée dans les villae méridionales et elle correspond généralement à des installations vinicoles (Brun, 1993a, p. 307 sqq.). À Bapteste, il est difficile d'aboutir à une certitude car on n'a découvert qu'une seule empreinte éventuelle de dolium, la fouille n'ayant pas encore été étendue vers le sud-ouest où le chai pourrait se trouver. 


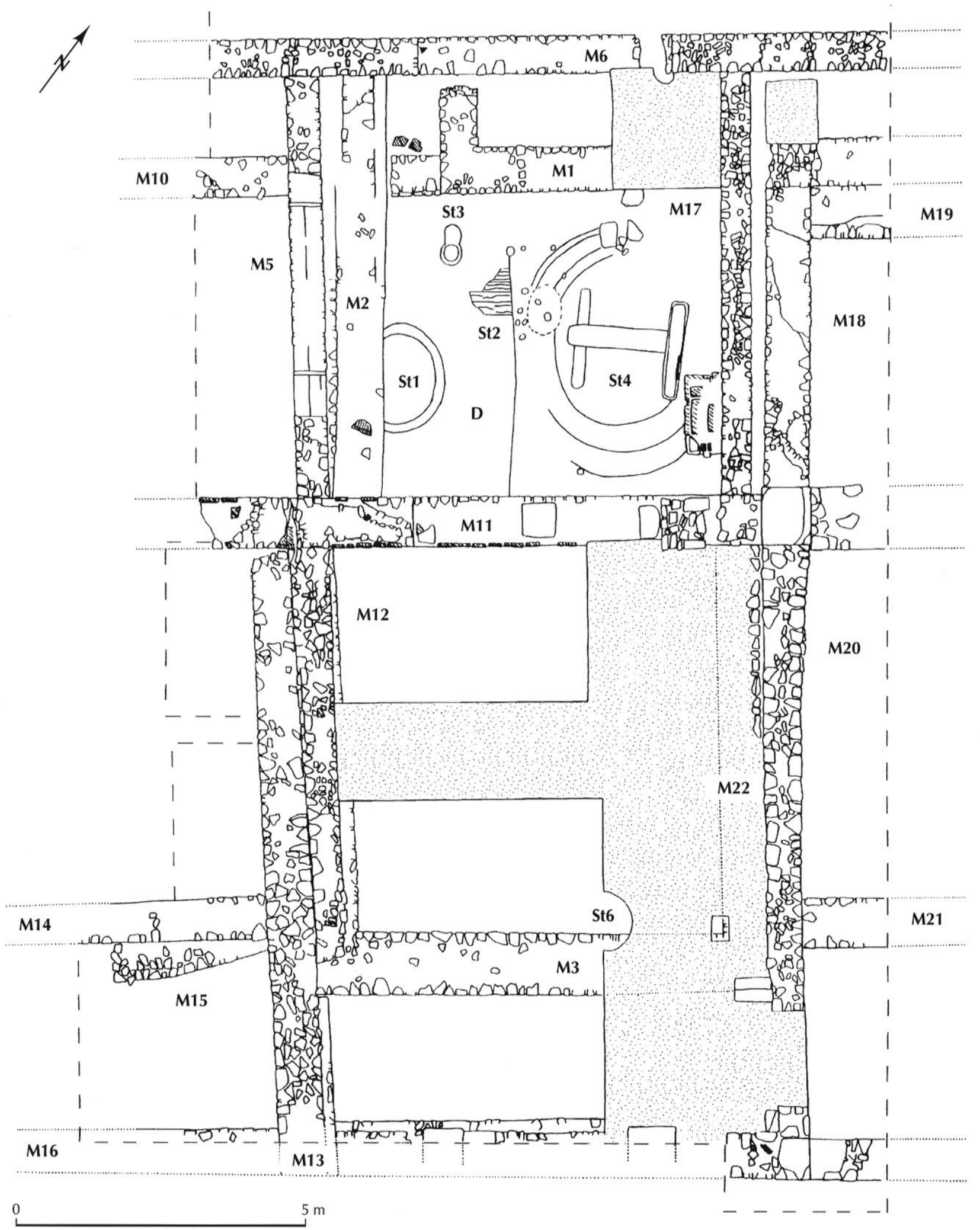

Fig. 64 - Plan des installations agricoles de l'état 2 de la villa de Bapteste à Moncrabeau, Lot-et-Garonne (relevé P. Jacques, 1996). 


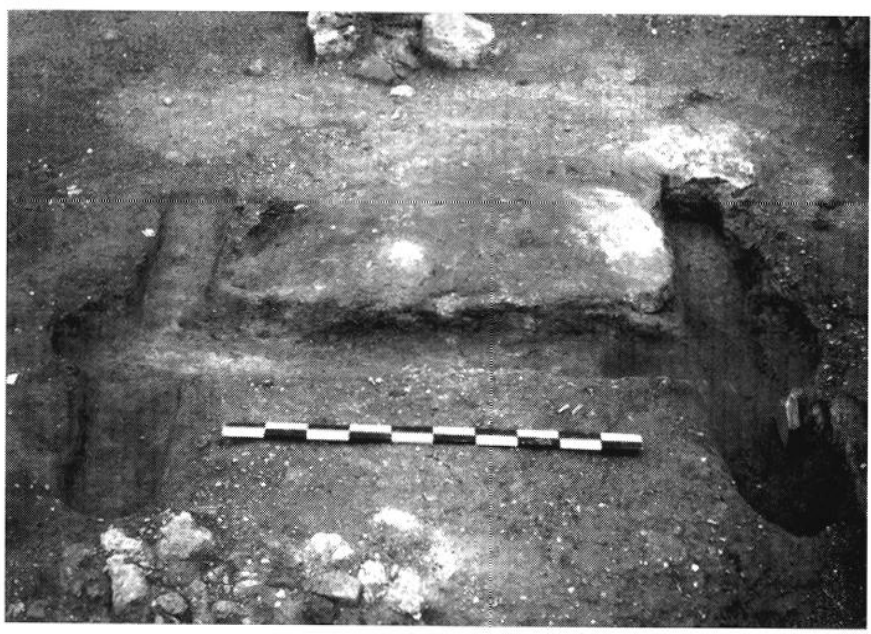

Fig. 65 - Empreinle laissée dans l'argile par le socle du pressoir de la villa de Bapteste à Moncrabeau, Lol-el-(xaronne (cliché r. Jacques, 1996).

L'implantation de ce bâtiment est datable du début du II' s. La durée de vie de cette installation ne semble pas avoir excédé un siècle.

Somme toute, l'élément majeur de cette intervention a été la misc en évidence d'un pressoir à vin sans doute lié à une aire de stockage de dolia; il s'agit de la première trace d'installation viticole découverte à ce jour en Lot-ctGaronne. Il importe en outre de souligner que les fouilles du XIX's. ont livré, parmi l'abondant mobilier lić à l'agriculture, plusieurs serpettes à vendanger (actuellement exposées au musée de Nérac). Ces dernières proviennent de l'état de la fin de l'Antiquité. Elles semblent confirmer la tradition viticole de ce domaine pendant une grande partie de l'Empire.

P. J.

\section{Belmont à Royan (Charente-MaRitime)}

En 1999, les travaux de la rocade de contournement de Royan ont été précédés d'interventions archéologiques conduites par l'Association des fouilles archéologiques nationales (AFAN). Au lieu-dit Belmont, sur le bord d'un plateau calcaire, se situaient les bâtiments, très arasés, d'une exploitation agricole d'époque romaine dominant, à $22 \mathrm{~m}$ d'altitude, l'ancien rivage de la Gironde. Deux corps de bâtiments étaient disposés en " $\mathrm{L}$ ». À l'ouest, une aile d'habitation mesurant $30 \mathrm{~m}$ sur $13 \mathrm{~m}$ au moins comprenait une demi-douzaine de pièces qui connurent deux phases. Au sud, deux bâtiments délimitaient une cour ouverte vers le nord et l'est.
Le bâtiment 2 abritait une installation vinicole (fig. 66 et 67 ) et le bâtiment 3 pourrait être un grenier.

Le chai, long de $28 \mathrm{~m}$ et large de $17 \mathrm{~m}$, était divisé en trois nefs. La nef centrale comportait à l'est un espace mesurant $7 \mathrm{~m}$ sur $3,80 \mathrm{~m}$ où se situaient vraisemblablement deux fouloirs et un pressoir puisque trois cuves sont accolées à son mur ouest. Ces cuves, construites en une seule fois, étaient ceinturées par une maçonnerie de moellons et de fragments de tegulae, recouverte intérieurement d'un enduit de mortier de tuileau. Le fond des cuves était constitué d'une couche de mortier de tuileau reposant sur un hérisson de pierres calcaires. Des boudins en quart-de-rond rendaient étanches les angles du fond et des parois (fig. 68). Dans l'angle sud-est des cuves nord et centrale se trouvaient les restes d'un emmarchement au pied duquel s'ouvrait une large cuvette de vidange. La superposition de trois à cinq couches de mortier montre que les fonds des cuves furent réparés à plusieurs reprises, ce qui implique une longue durée d'utilisation. Le niveau supérieur était constitué

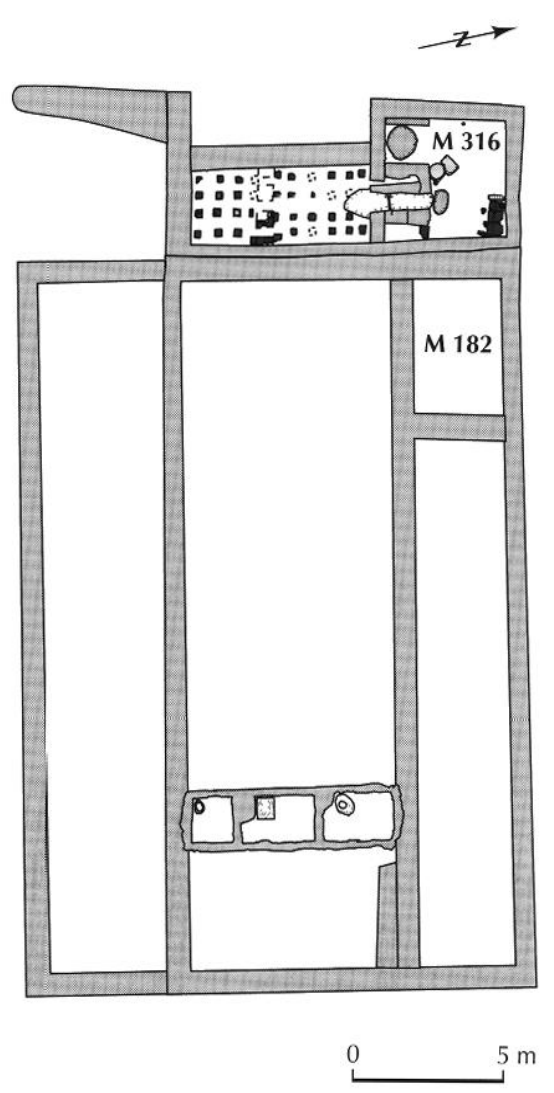

Fig. 66 - Plan d'ensemble du bâtiment vinicole de Belmonı à Royan, Charente-Maritime (dessin K. Robin, 1999). 


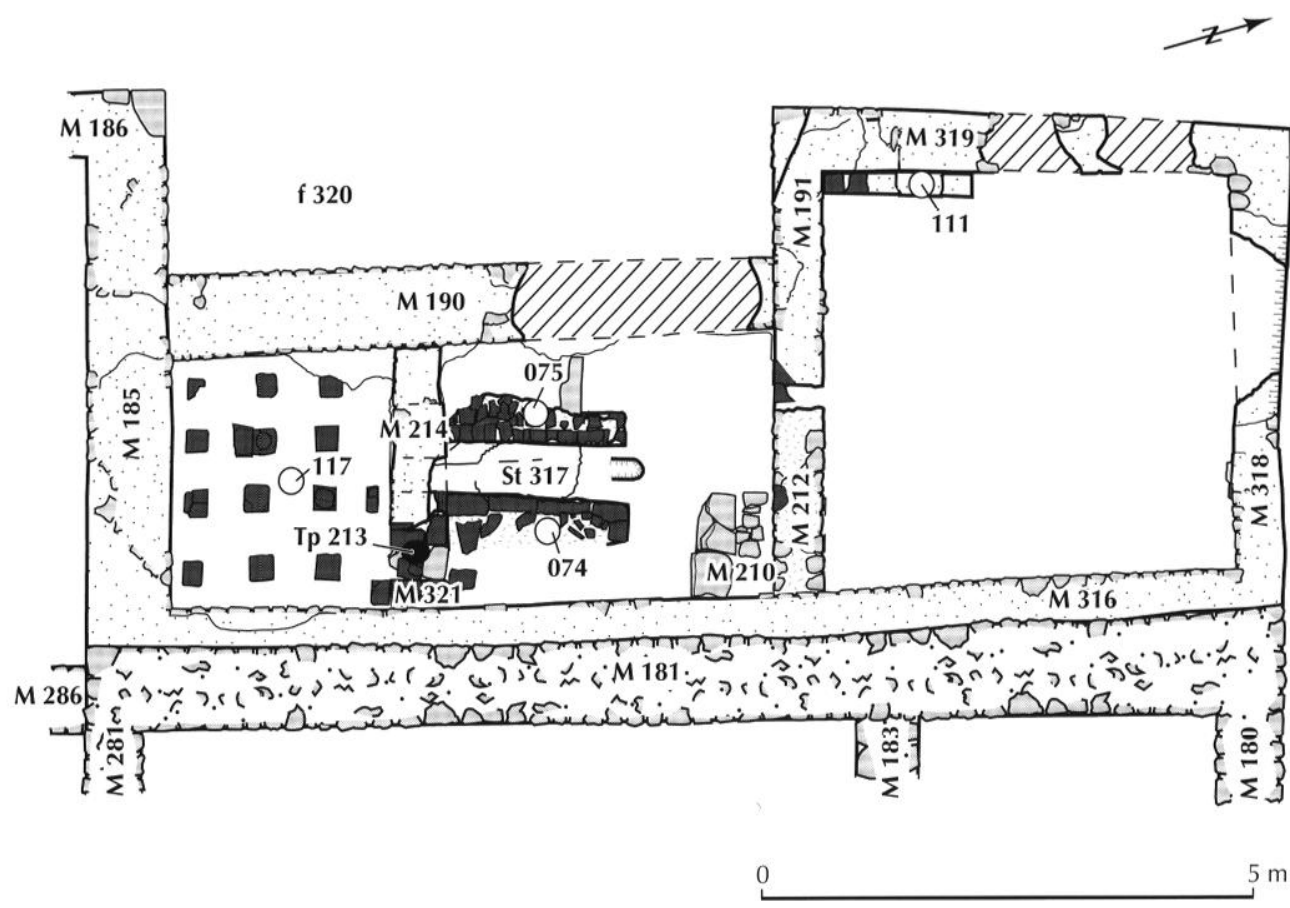

Fig. 67 - Relevé de la seconde phase de la salle chauffée à l'ouest du bâtiment de Belmont à Royan, Charente-Maritime. (relevé K. Robin, 1999).

non d'une forme en béton mais de dalles de calcaire tendre, amincies par des raclages intensifs. Le reste de la nef centrale et la nef septentrionale pourraient avoir servi à entreposer les vaisseaux vinaires, probablement des foudres de bois. La nef méridionale correspondait soit à une extension du chai, soit à une galerie ouverte.

Un praefurnium et une pièce sur hypocauste accolés à la façade ouest du bâtiment semblent participer à la fois de la vie de la ferme et de l'utilisation du chai. Cet ensemble a évolué au cours du temps : deux phases principales sont décelables. Dans un premier temps, le praefurnium chauffait une pièce longue de $6 \mathrm{~m}$ et large de 2,30 m (fig. 66). L'importance des maçonneries situées de part et d'autre de la chambre de chauffe montre qu'elle supportait une ou deux chaudières. Dans un second état (fig. 67), la salle sur hypocauste fut réduite à un carré de $2 \mathrm{~m}$ de côté environ et un nouveau foyer, supportant lui aussi une chaudière, fut implanté au milieu de l'ancienne salle chaude. Cette installation ne peut être assimilée à des thermes; il n'y a point de frigidarium ni de tepidarium et un baigneur de qualité ne pouvait donc effectuer le parcours classique. On doit plutôt la rapprocher de structures similaires découvertes à Péré Maillard à Soubise, et dans plusieurs chais de la vallée de la Moselle (à Piesport et à Erden, par exemple).

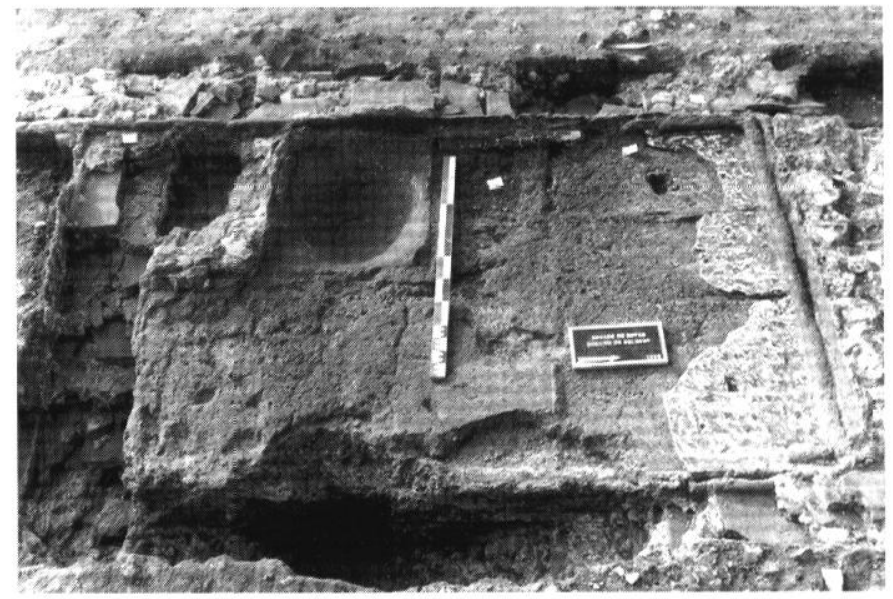

Fig. 68 - Cuve de recueil du mô̂t de Belmont à Royan, Charente-Maritime (cliché K. Robin, 1999).

Il s'agirait de fumaria, ces apothèques où l'on accélérait le vieillissement des vins en chauffant amphores et tonneaux. Dans le cas de Belmont, il est possible que, comme le recommande Columelle (De l'agriculture, I, $6,20)$, ces pièces chauffées aient aussi servi accessoirement de thermes rustiques pour la main-d'œuvre. Les chaudières placées sur le foyer servaient probablement à la cuisson du defrutum, ce jus de raisin concentré dont 
l'adjonction au moût en cours de fermentation garantissait l'élévation du degré alcoolique et une meilleure conservation du vin.

La datation du bâtiment agricole de Belmont est rendue délicate par son état d'arasement. Deux fosses, liées au premier état de la ferme, contiennent du mobilier datable du Haut-Empire et il est vraisemblable que l'habitation, le chai et les premières cuves ont été édifiés à cette période. L'essentiel du mobilier trouvé dans les couches d'occupation des pièces chaudes, notamment dans leur second état, est, quant à lui, attri- buable aux $\mathrm{III}^{\mathrm{e}}$ et $\mathrm{IV}^{\mathrm{e}} \mathrm{s}$. : le dernier état de la pièce chaude et des cuves, en particulier le dallage de calcaire, doit dater du IVes.

K. R.

\section{Les Chapelles à Port-des-Barques (Charente-Maritime)}

Le site des Chapelles a fait l'objet, en 1999, d'une évaluation archéologique qui a permis de dessiner les contours d'une villa complète, associant des bâtiments

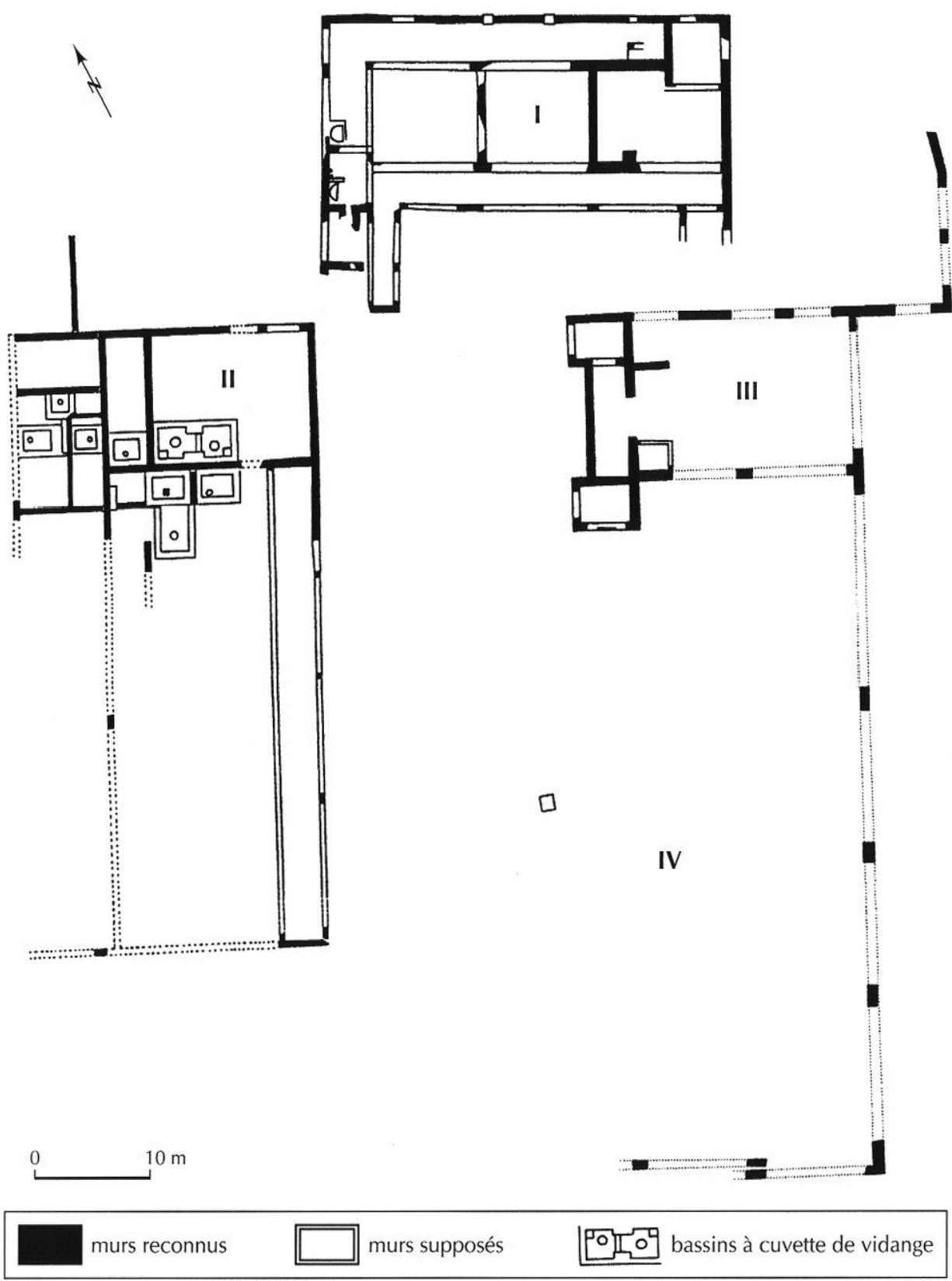

Fig. 69 - Plan d'ensemble de la villa des Chapelles à Port-desBarques, Charente-Maritime: à l'ouest (bâtiment II), l'installation vinicole (dessin P. Duprat, 1999). 
résidentiels et des structures de type agricole (fig. 69). Trois corps de bâtiments (I-II-III) délimitent un vaste espace non construit (IV) : au nord, une zone d'habitation (I) ; à l'ouest, des locaux à destination vinicole partiellement reconnus en 1987-1988 (II) ; à l'est, des bâtiments agricoles non définis (III).

Le bâtiment I est constitué d'un quadrilatère divisé en trois espaces, auquel se greffe une sorte de galerie périphérique. Au centre de la façade nord, deux pierres cubiques sont les vestiges probables d'une structure d'entrée, bouchée plus tard par un mur. La galerie est coupée au nord-est par un bassin (ou une citerne), et, au sud-ouest, par un balnéaire comportant au moins deux baignoires, dont l'une sur hypocauste. La structure originelle est datable de la fin du ${ }^{\text {er }}$ s., les adjonctions s'étalant jusqu'au III' $s$. (galerie), voire au IV (balnéaire).

L'ensemble II forme un vaste bâtiment ( $50 \mathrm{~m}$ x $25 \mathrm{~m}$ ) qui se caractérise par l'existence de bassins liés à des aires de travail, et par la présence de chais. Ces bassins, peu profonds ( $1 \mathrm{~m}$ environ), étanches et dotés de cuvettes de vidange, collectent le liquide élaboré sur des aires étanches qui leur sont associées. Les salles oblongues, au sol en terre battue, sont probablement des chais. Dans un premier temps, cinq bassins (dont l'un dédoublé) sont adossés à des murs datés de la fin du I ${ }^{\mathrm{er}}$ s. Ce complexe est ultérieurement agrandi à l'ouest (trois bassins et aires supplémentaires).

Les bâtiments agricoles III, à peine entrevus au cours de l'évaluation, sont des constructions frustes liées à un long mur extérieur $(70 \mathrm{~m})$, très dégradé, peut-être un mur de clôture. L'espace IV se présente comme une cour au sol de galets tassés, avec une couche d'occupation datable de la fin du III' $s$. Vers le centre, le hasard des sondages a mis en évidence une énorme pierre taillée $(1,30 \mathrm{~m} \times 1,15 \mathrm{~m} \times 0,35 \mathrm{~m})$ reposant sur un socle maçonné, dont on ignore le rôle.

Le site ne semble plus fonctionner comme exploitation viticole aux $\mathrm{V}^{\mathrm{e}}-\mathrm{VI}^{\mathrm{e}} \mathrm{s}$. (comblement des bassins les plus tardifs). Le bâtiment II est réoccupé durant le haut Moyen Âge. Par ailleurs, plusieurs sépultures, apparemment médiévales, parsèment les structures (I-II-IV). À quelques kilomètres, la villa du Renfermis (Soubise), actuellement détruite, présentait la même conception architecturale (bâtiments d'habitation liés aux locaux viticoles).

\section{La Fontaine à Reignac (Charente)}

Un sauvetage urgent réalisé sur le tracé de la déviation de Barbezieux-Saint-Hilaire en 1994 a mis au jour, au lieu-dit La Fontaine, des traces d'enclos et de culture d'époque romaine, couvrant $22500 \mathrm{~m}^{2}$. Deux parcelles d'une surface minimale de $1300 \mathrm{~m}^{2}$, délimitées par trois fossés parallèles est-ouest, ont été reconnues (fig. 70). La parcelle sud présente 117 tranchées rectangulaires, orientées nord-sud, longues de $1 \mathrm{~m}$ à $1,30 \mathrm{~m}$ et larges de $0,10 \mathrm{~m}$ à $0,15 \mathrm{~m}$, pour une profondeur conservée de $0,10 \mathrm{~m}$ à $0,20 \mathrm{~m}$, les extrémités étant plus profondément creusées. Ces tranchées, espacées de $1,10 \mathrm{~m}$, sont disposées en 6 rangées dans le sens nord-sud et en 28 au moins dans le sens est-ouest. Dans la parcelle nord, plus érodée, 43 tranchées ont été observées. Analogues aux précédentes quant aux dimensions, elles en diffèrent par l'orientation est-ouest. L'analyse palynologique des prélèvements effectués au fond des tranchées n'a donné aucun résultat, mais on peut envisager l'hypothèse qu'il s'agisse de traces de plantation de vignes.

$\mathrm{Au}$ nord-est de cette parcelle, en limite de la fouille, se trouvait un bassin carré mesurant $1 \mathrm{~m}$ de côté et conservé sur $1 \mathrm{~m}$ de profondeur. Les parois, construites en fragments de tegulae, étaient recouvertes d'un enduit de mortier de tuileau, avec des joints d'étanchéité aux arêtes. Le fond du bassin comportait une cuvette de vidange mesurant $0,26 \mathrm{~m}$ de diamètre. Les niveaux de sols correspondant à ce bassin avaient été emportés par l'érosion.

On ne possède aucun élément permettant de dater le creusement des tranchées de culture et la construction du bassin, mais on remarque que des fosses d'extraction de calcaire recoupant les tranchées sont comblées avec du mobilier du II $^{e} s$. Par ailleurs, le comblement du bassin contient du matériel de la fin du $\mathrm{III}^{\mathrm{e}} \mathrm{s}$. Si le remplissage de la cuve intervient à cette période, il faudrait peut-être rapprocher cet événement de l'enfouissement d'un dépôt monétaire de 1937 monnaies de Caracalla à Salonin.

Deux puits, qui ont été découverts à proximité, ont livré des gravats antiques: tegulae, mortier, moellons, éléments de statue. Ils indiquent probablement l'existence d'une villa à laquelle pourraient appartenir le bassin et d'éventuelles structures complémentaires situées plus à l'est, au-delà de la zone fouillée. K. R.

P. D. 


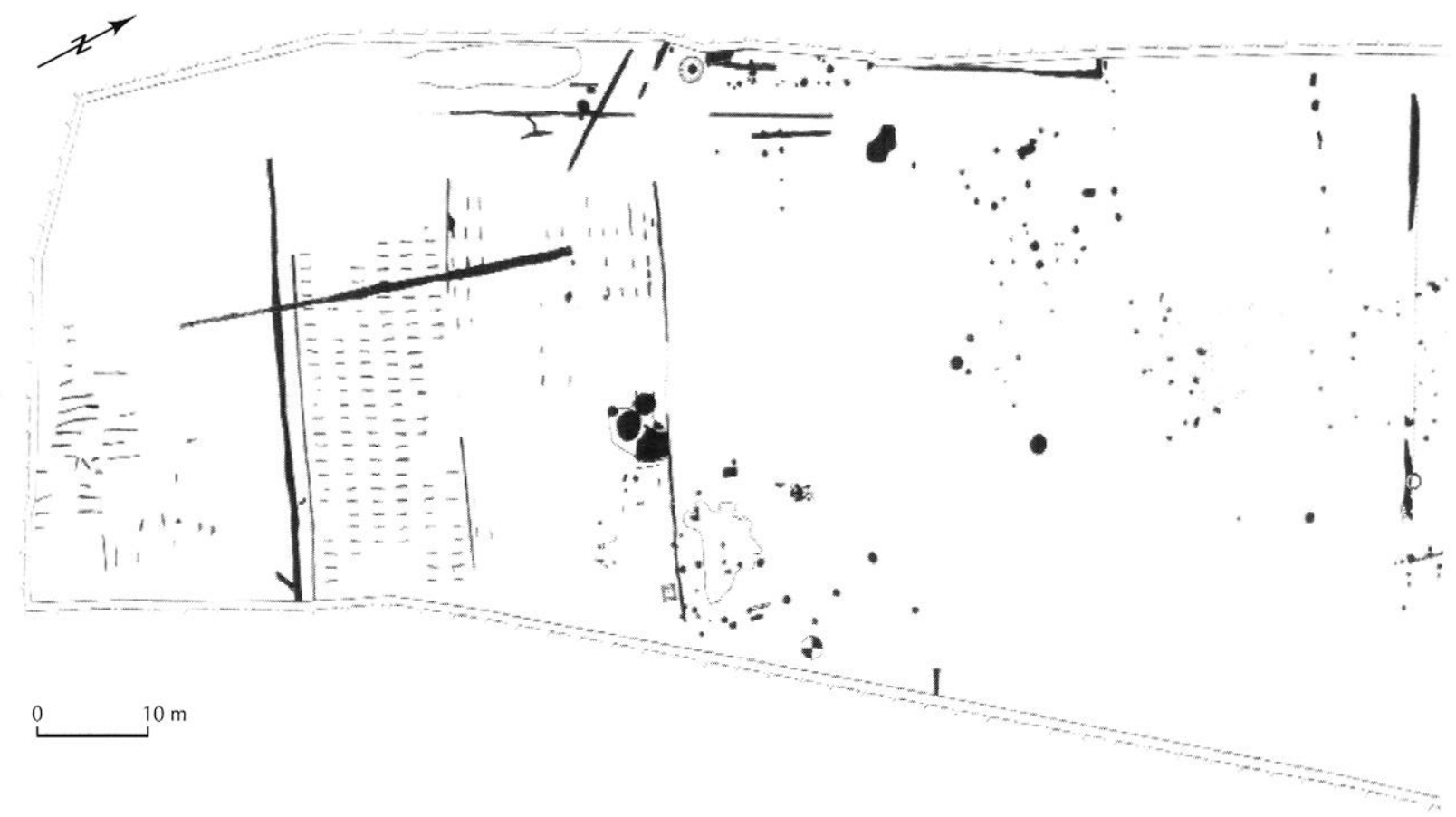

Fig. 70 - Relevé des tranchées de culture de La Fontaine à Reignac, Gironde : au nord-est un petit bassin à cuvette de vidange (dessin K. Robin, 1994).

\section{La Haute-Sarrazine à Cognac (Charente)}

En avril 1987, une fouille préventive effectuée dans la périphérie nord-ouest de la ville de Cognac (au lieu-dit La Haute-Sarrazine) a permis de dégager dans leur totalité les bâtiments d'une exploitation agricole d'époque antique de grande envergure, organisée en deux ailes disposées à angle droit (Gallia Informations, 1989-2, p. 245-246).

L'aile A mesure $80 \mathrm{~m}$ de long sur $16 \mathrm{~m}$ de large avec un mur de refend longitudinal, l'aile B $35 \mathrm{~m}$ de long sur $11 \mathrm{~m}$ de large. Une petite pièce rectangulaire 16 a été construite dans l'angle interne des deux bâtiments. À l'est de l'aile B, se développe une vaste cour limitée par des murs mal fondés (fig. 71). La pars urbana de cette villa n'a pas été reconnue avec certitude. L'observation de murs maçonnés et la découverte de monnaies antiques dans les jardins d'une zone pavillonnaire, à une centaine de mètres au sud-est, peuvent être des indices à confirmer.

Dans la zone formant la jonction entre les deux ailes A et $B$, plusieurs bassins maçonnés ont été mis au jour. Leur nombre impressionne, mais ils n'ont jamais fonctionné tous en même temps (Vernou et al., 1990, p. 29-32). On note toutefois des caractéristiques identiques : parois constituées de fragments de tegulae liés au mortier de chaux, enduit de surface étanche constitué d'un mortier mêlant chaux, sable fin et brisure de briques, joints d'ćtanchéité dans tous les angles et cuvette de vidange aménagée dans le fond du bassin (fig. 72). Certains, qui fonctionnaient par paire, étaient reliés à leur base par des tuyaux de plomb d'un diamètre intérieur de $5 \mathrm{~cm}$ (par ex.: bassins 10 et 11). Leurs dimensions, variables, sont détaillées dans la publication (Vernou et al., 1990, p. 23).

Immédiatement après cette découverte, ces vestiges n'avaient pas été interprétés de manière assurée, même si l'hypothèse de l'activité vinicole avait été évoquée (Vernou el al., 1990, p. 37). Le réexamen attentif de l'architecture incite désormais à identifier l'ensemble comme une installation vinicole. La présence de pépins de raisin et la découverte de serpettes confortent du reste cette hypothèse. Les bassins en batterie décrits plus haut devaient recevoir le jus de raisin provenant d'une aire de foulage attenante. L'espace 7 du bâtiment $B$, remarquable par ses vastes dimensions $(8 \mathrm{~m} \times 5,50 \mathrm{~m})$, devait être le fouloir. Il a connu au moins deux rechapages successifs de mortier de tuileau, indiquant une certaine 


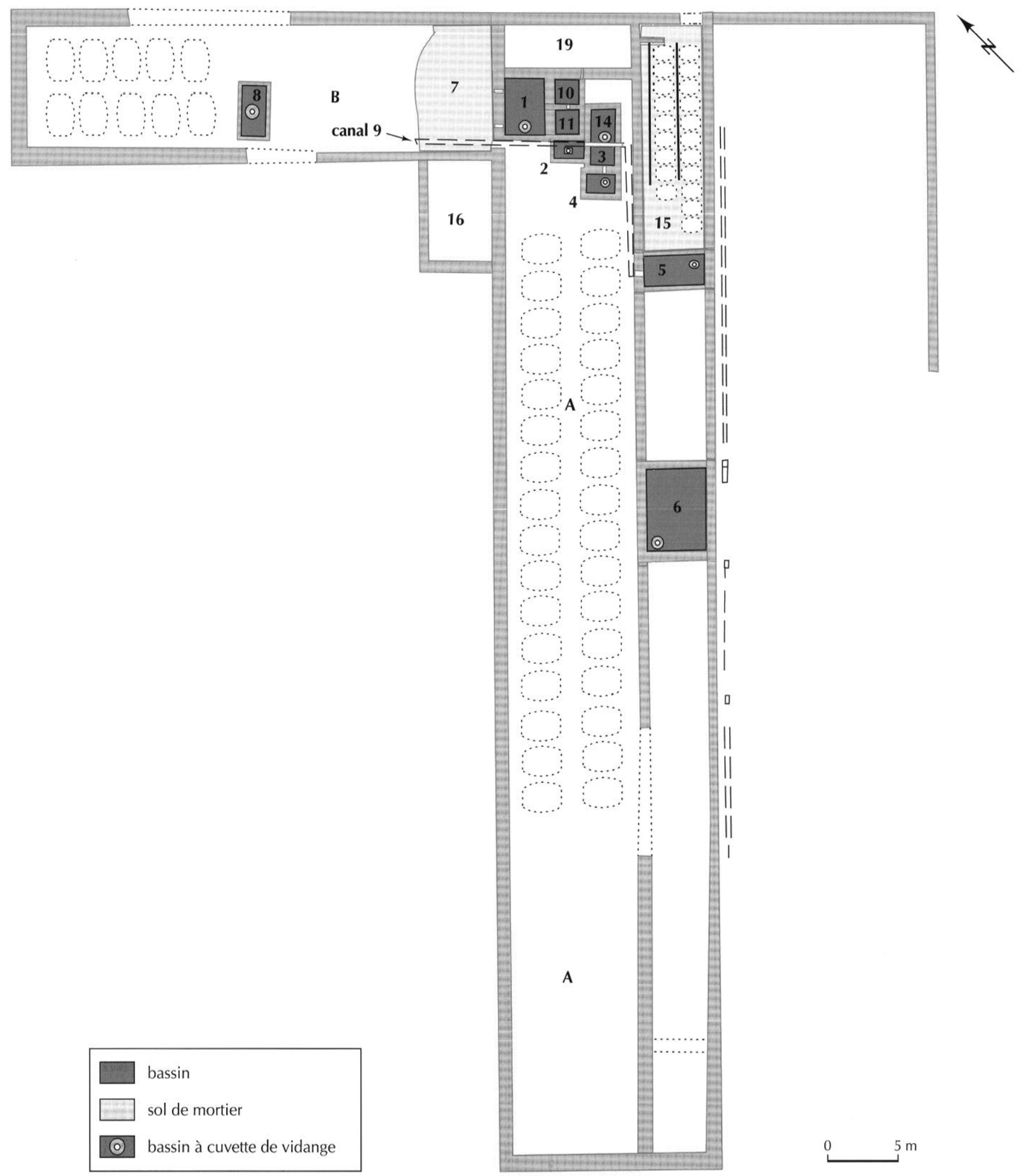

Fig. 71 - Plan de l'établissement vinicole de La Haute-Sarrazine à Cognac, Charente (dessin H. Gaillard, 1999, d'après Vernou et al., 1990). 


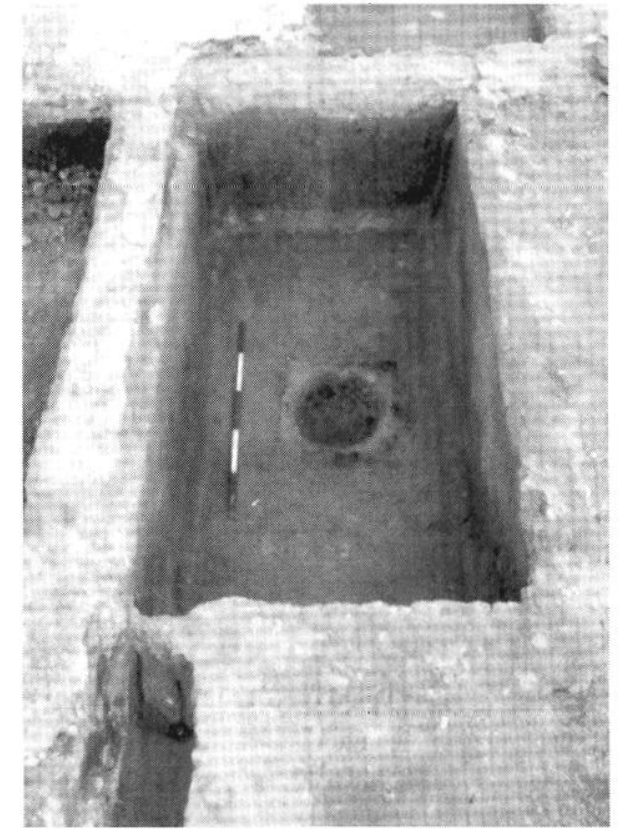

Fig. 72 - La Haute-Sarrazine à Cognac, Charente : bassin de recueil du mô̂t avec sa cuvette en pierre, vu du nord (cliché C. Vernou).

longévité de la production vinicole. Dans un premier temps, le fouloir devait alimenter les bassins jumeaux 10 et 11 par deux tuyaux de plomb. Par la suite, le bassin 1 est constitué au détriment des bassins jumeaux, mais le mode d'alimentation perdure. Notons pour information que le bassin 1 a une contenance de plus de $15 \mathrm{~m}^{3}$, soit $150 \mathrm{hl}$, ce qui est considérable. Peut-être de manière contemporaine, le jus provenant du fouloir pouvait être dirigé dans un petit canal 9 formant rigole et sc jetant dans le bassin 5 . On ne connait pas le fouloir qui alimentait les bassins 3-14-4, ni celui du bassin 8 qui se trouve isolé dans le bâtiment B. Quant au bassin 6, se situant à l'ouest du bassin 5, il était peu profond : moins de $30 \mathrm{~cm}$ au-dessous du niveau de circulation. Son interprétation demeure incertaine.

À titre d'hypothèse de travail, il est tentant de voir dans l'espace 19 une aire où auraient pu se situer un ou plusieurs pressoirs en bois à vis verticale. Dans cette zone, aucun sol n'a été observé, mais il subsistait un hérisson irrégulier de moellons calcaires non équarris. Étaient-ce les vestiges d'un massif maçonné ? Dans ce cas de figure, la rafle récupérée dans le fouloir 7 était transposée dans les pressoirs de la zone 19 et le jus obtenu pouvait s'écouler dans le bassin 11, puis dans le bassin 1.
Comme on le sait, le moût était retiré des citernes avant la fermentation. À Cognac, comme sur la plupart des sites du Grand Sud-Ouest, aucun dolium n'a été découvert. Il faut donc envisager que les tonneaux devaient jouer le rôle de contenant. Le secteur 15 pouvait correspondre à un chai réservé à la fermentation de vins spécifiques. Son sol était étanche et deux rigoles rectilignes étaient aménagées en direction du bassin 5 , solution commode pour récupérer le vin en cas de fuite.

Les vastes espaces vides de tout aménagement à l'intérieur des bâtiments $\mathrm{A}$ et $\mathrm{B}$ devaient correspondre à des zones de vinification où se trouvaient tonneaux et foudres en chêne des forêts de Saintonge. Les capacités de vinification comme les dimensions du fouloir font du site de La Haute-Sarrazine une exploitation vinicole exceptionnelle.

On manque d'éléments stratigraphiques pour dater la construction de l'établissement. De la nature et de la qualité des maçonneries, on peut toutefois inférer une datation à partir de la seconde moitié du $\mathrm{I}^{\mathrm{er}} \mathrm{s}$. ou dans le courant du II's. Pour l'abandon, un terminus ante quem est donné par le mobilier découvert dans le comblement des bassins 8 et 5 : le dernier quart du $\mathrm{III}^{\mathrm{e}} \mathrm{s}$. (Vernou et al., 1990). Parmi les dizaines d'outils en fer dégagés dans le bassin 5, quelques serpettes de vigneron ont été identifiées (voir étude ci-dessous). Les derniers habitants des lieux ont pu entretenir une activité vinicole limitée et le bassin 2, le plus tardif, avec sa cuvette de vidange en pierre, a pu servir aux ultimes productions. En partie supérieure de son comblement, la découverte d'un follis constantinien peut indiquer la période d'abandon définitif du site (Vernou et al., 1993, p. 118-120).

C. V.

\section{Lestagnac à SaInt-Mézard (Gers)}

Une prospection aérienne, effectuée en 1995, a révélé le plan complet de la villa de Lestagnac, située à mi-pente du versant exposé au sud d'un vallon de rive gauche de la vallée du Gers. Cet établissement rural assez vaste (3 $300 \mathrm{~m}^{2}$ environ) comporte deux ensembles de bâtiments accolés. Le premier, au sud-sud-ouest, qui est formé de deux corps disposés en « $\mathrm{L}$ », constitue la pars urbana de la villa. Le second, au nord-nord-est, est un très grand édifice orienté est-ouest, d'environ $75 \mathrm{~m}$ de long sur $15 \mathrm{~m}$ de large, bordé d'une galerie de $5 \mathrm{~m}$ de large 


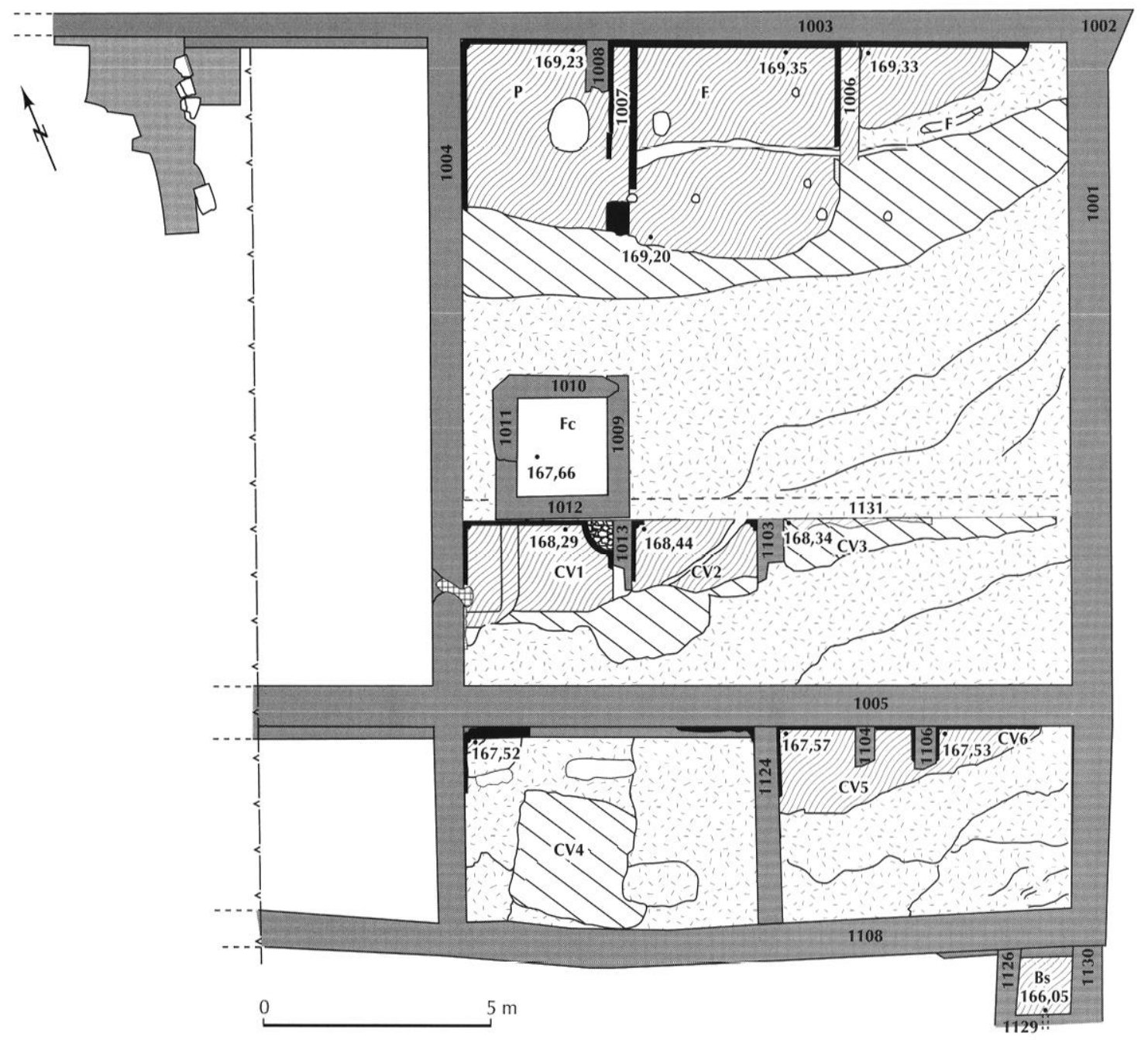

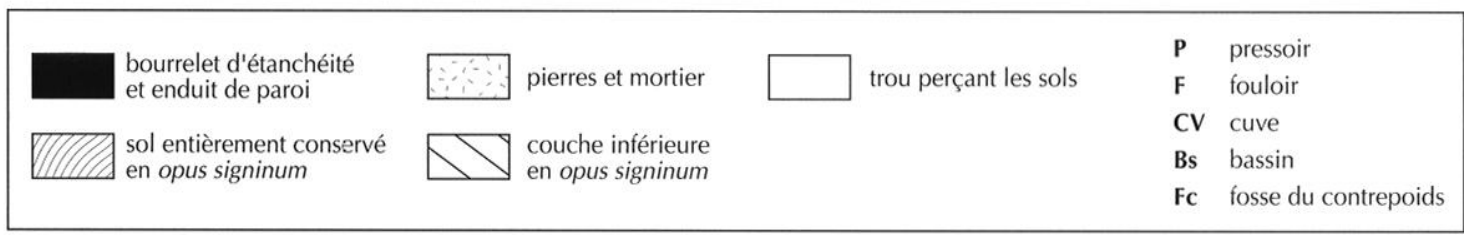

Fig. 73 - Relevé du bâtiment vinicole de la villa de Lestagnac à Saint-Mézard (Gers), partiellement mis au jour en $1999-2000$ (dessin J.-M. Labarthe, M. Fincker, V. Picard, CNRS, IRAA, 2000).

sur son côté sud (fig. 73). Ce long bâtiment est divisé en deux parties seulement qui ont également de grandes dimensions: l'une, à l'ouest, d'environ $60 \mathrm{~m}$ de longueur ; une seconde, à l'est, de $15 \mathrm{~m}$ de côté environ, dont l'aspect blanchâtre sur la photographie aérienne révèle un sol à revêtement épais, en pierres ou en béton. La place de cet édifice par rapport au corps résidentiel, ses dimensions imposantes et l'absence de petites pièces invitent à l'identifier comme le bâtiment d'exploitation de la villa. L'hypothèse d'une installation vinicole a été formulée pour expliquer le sol en dur de son extrémité orientale (Petit, 1997, vol. 1, p. 185).

Afin de vérifier ces hypothèses, un premier décapage a été effectué en 1999 dans cette partie est du bâtiment : malgré le fort arasement des structures antiques, il a permis de confirmer que l'on était effectivement en présence d'une cella vinaria au sol entièrement revêtu de béton de tuileau. Ensuite, en raison de l'intérêt de cette villa viticole de l'Aquitaine méridionale, une fouille de l'ensemble du bâtiment d'exploitation a été program- 
mée : en 2000, la salle de vinification a été entièrement mise au jour.

L'équipement vinicole comptait un pressoir, vraisemblablement deux fouloirs et une batterie de six cuves. La moitié nord de la salle, qui est subdivisée en trois compartiments par des murets nord-sud, rassemblait les installations de pressurage et de foulage disposées parallèlement. Bien qu'il ne subsiste aucun vestige du pressoir, celui-ci est clairement identifié, grâce à la présence d'une fosse maçonnée destinée à recevoir le contrepoids du praelum: il se trouvait dans le compartiment ouest, large de $3,20 \mathrm{~m}$, à sol de béton de tuileau légèrement incliné vers le sud et pourvu de bourrelets périphériques d'étanchéité. Comme il n'existe pas de trace des jumelles, la tête du praelum était sans doute encastrée dans le mur nord du bâtiment. Ensuite, à l'est du pressoir, se trouvaient très probablement deux fouloirs semblables: chacun était large de $4,50 \mathrm{~m}$ et avait un sol revêtu de béton de tuileau, également en pente vers le sud et bordé de quarts-de-rond d'étanchéité ; leur longueur était sans doute de 9,50 m environ, car ils occupaient vraisemblablement tout l'espace compris entre le mur nord du bâtiment et le premier alignement de cuves.

Deux rangées de cuves, installées sur la partie sud de la salle et toute la largeur de la galerie, se succèdent ensuite. Elles sont très arasées, puisqu'elles ne conservent généralement qu'un lambeau de leur revêtement de fond (ce qui explique probablement l'absence de cuvette de vidange). La première rangée, immédiatement au sud du pressoir et des fouloirs, comporte deux cuves de taille moyenne à l'ouest $(3,30 \mathrm{~m} \times 3,40 \mathrm{~m} ; 2,80 \mathrm{~m} \times 3,40 \mathrm{~m})$ et une nettement plus grande à l'est $(6,20 \mathrm{~m} \times 3,40 \mathrm{~m})$. La cuve de l'ouest (au sud du pressoir) est un peu moins arasée que les autres: la base en quart de cercle d'un petit escalier d'angle y subsiste ainsi que le départ d'un conduit de vidange qui traversait le mur séparant la salle de vinification du chai. Les trois cuves de la seconde rangée présentent les mêmes différences de taille, mais leur disposition est inversée puisque la grande $(6,30 \mathrm{~m}$ x $4 \mathrm{~m}$ ) est placée à l'ouest et les deux moyennes $(3 \mathrm{~m}$ x $4 \mathrm{~m}$ ) à l'est. Flles sont aussi nettement en contrebas par rapport aux autres, leur sol se trouvant à environ $80 \mathrm{~cm}$ (cotes $167,52 / 167,57$ ) au-dessous de celui des précédentes (cotes 168,29/168,44). Enfin, un petit bassin de forme carrée (1,20 m de côté), également revêtu de mortier de tuileau, est accolé à la face sud du bâtiment, à l'angle sud-est. Son sol est encore plus bas d'une cinquan- taine de centimètres (cote 166,05). L'usure et souvent l'arasement total d'une grande partie des sols ayant entraîné la disparition de toutes les rigoles et conduits de circulation du moût, il est particulièrement difficile de définir la destination des différentes cuves et le fonctionnement de l'ensemble : la disposition en deux niveaux révèle peut-être cependant une phase de décantation dans le processus de vinification.

On a également commencé la fouille du chai, situé à l'ouest de la salle de vinification. Cette partie du bâtiment est tout autant arasée et son sol en terre battue ne subsiste que le long du mur nord.

En raison de très importants bouleversements occasionnés par les engins aratoires, la chronologie du site est encore incertaine. Pour le moment, on se bornera à indiquer que la cella vinaria était en fonctionnement au Haut-Empire et qu'une réoccupation de son bâtiment a eu lieu au IV $\mathrm{V}^{\mathrm{e}} \mathrm{s}$., mais à des fins autres que vinicoles.

C. P.-A. et P. S.

\section{La Pointe des Minimes à La Rochelle (Charente-Maritime)}

Entre 1979 et 1982, la Société d'archéologie et d'histoire de l'Aunis eut le grand mérite de fouiller une villa romaine en cours de destruction par les travaux de voirie d'un lotissement. Les travaux, dirigés par J. Flouret et G. Durand, ont montré que les vestiges couvraient environ 1 ha et s'inscrivaient pour l'essentiel dans un grand rectangle délimité par des murs de clôture (fig. 74). À l'ouest, se trouvait la pars urbana comprenant des thermes et plusieurs pièces chauffées par des hypocaustes à canaux. À l'est, la pars fructuaria, en partie détruite par les travaux de voirie, s'étendait sur $4000 \mathrm{~m}^{2}$ au moins.

Selon les fouilleurs, les premiers bâtiments de la villa ont été édifiés durant le dernier tiers du $\mathrm{I}^{\mathrm{er}}$ s. après J.-C., époque à laquelle ils attribuent la construction de thermes décorés de peintures ainsi que celle de bâtiments agricoles comportant des bassins plusieurs fois remaniés (état 1). Dans la seconde moitié du III $\mathrm{e}$., peutêtre après un abandon temporaire (état 2) ${ }^{173}$, des trans-

173. Les auteurs de la publication supposent un abandon dans la première moitié du $\mathrm{III}^{\mathrm{e}} \mathrm{s}$. car ils constatent une lacune dans les séries monétaires. Cet argument n'est guc̀re convaincant, car les monnaies des Sévère sont toujours plus rares que les autres. 
Fig. 74 - Plan de linstallation vinicole de La Pointe des Minimes à La Rochelle, Charente-Maritime (dessin H. Gaillard, 1999, d'après le plan de A. Bergé).

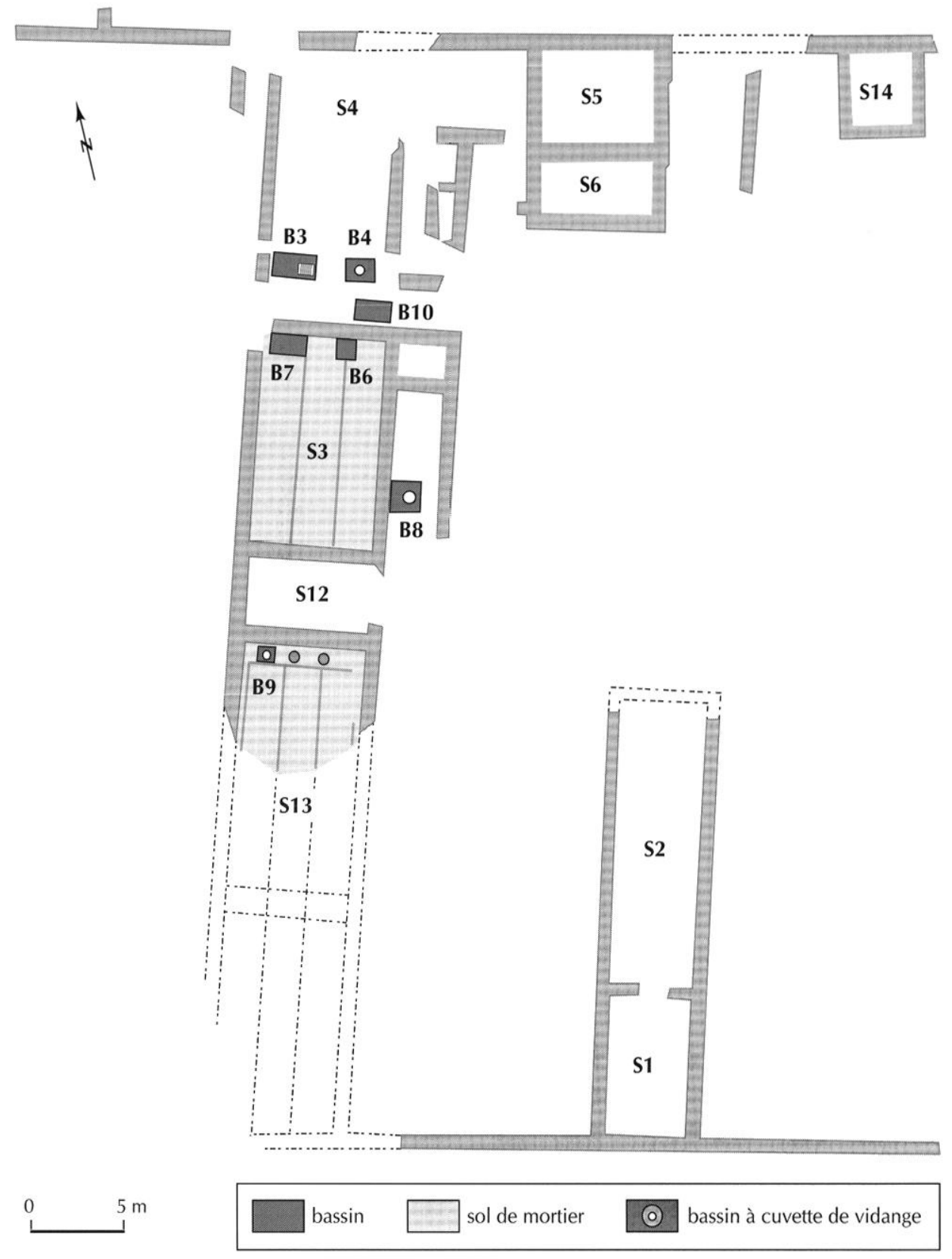

Un état 4 , datable de la première moitié du IV ${ }^{\mathrm{c}}$ s., est marqué par un changement d'utilisation des bâtiments agricoles dont témoignent la constitution d'une couche d'occupation sur le béton de la salle S3 et le comblement du bassin B9 par un dépotoir.

Si l'on peut admettre cette chronologie relative dans ses grandes lignes, on est toutefois conduit à s'interroger sur telle ou telle interprétation. Il n'est pas évident que les thermes ne soient pas contemporains de la partie résidentielle, les hypocaustes à canaux correspondant à des pièces d'habitation chauffées et non à des thermes. De même, la n'a pas été réparée ensuite. 
succession des bassins est probablement à revoir. Les auteurs de la publication attribuent à la même période (Flaviens-Antonins) les cuves B3, B4, B5, B6, B7, B10 et $\mathrm{B} 11$. Or les cuves $\mathrm{B} 5$ et $\mathrm{B} 11$ (non représentées sur le plan) étaient situées à un niveau beaucoup plus élevé que les autres, ce qui rend leur utilisation conjointe improbable. Par ailleurs, les cuves B6 et B7 étaient construites à l'emplacement d'une cuve antérieure plus vaste non numérotée. Le seul point acquis concerne les bassins B6 et B7 qui étaient recouverts par la dalle bétonnée de la pièce S3 construite lors de l'état 3 . En revanche, il n'est pas assuré que les cuves B3 et B4 aient été détruites lors de l'état 3 (d'ailleurs, les auteurs ne l'affirment pas).

Au total, lors de l'état 3, on pourrait imaginer que fonctionnaient ensemble la salle S4, les cuves B3 et B4 et les salles S3 et S13. La pièce $\mathrm{S} 4$, dont le sol, surélevé, était totalement détruit, devait abriter les fouloirs et les pressoirs. L'espace compris entre la salle S4 et la salle S3 était en contrebas : entre les cuves, on a retrouvé les traces d'un escalier d'accès à la salle S4, comme c'est généralement le cas. Cet espace pourrait avoir servi aussi à loger les machineries des pressoirs, éventuellement à levier et vis. Les deux salles jumelles S3 et S13, séparées par une entrée $\mathrm{S} 12$ ouvrant sur la cour, présentent des dispositions caractéristiques des chais: sols bétonnés divisés en trois par des rigoles aboutissant, dans le cas de la salle $\mathrm{S} 13$ au moins, à un petit bassin $\mathrm{B} 9$ à cupule d'une contenance de 384 litres $(0,80 \mathrm{~m} \times 0,80 \mathrm{~m} \times 0,60 \mathrm{~m})$. Pour la salle S3, le dispositif pourrait avoir été légèrement différent et lié au bassin B8, que la publication ne mentionne pas. Ces deux pièces correspondent certainement aux chais proprement dits. De part et d'autre d'un couloir central devaient se trouver des files de foudres, les rigoles et les bassins servant à recueillir le vin s'échappant des récipients en cas de fuite.

La datation de l'état 3 dans la seconde moitié du III $^{\mathrm{c}} \mathbf{s}$. ne repose pas sur des bases fermes : le matériel trouvé dans les cuves B6 et $\mathrm{B} 7$ scellées par le béton de la pièce $\mathrm{S} 3$ n'est pas décrit et on ne peut guère croire à une phase d'abandon (état 2) qui serait seulement marquée par une lacune dans la série monétaire. L'état 3 pourrait aussi bien remonter au II $^{\mathrm{e}} \mathrm{s}$. ou au début du $\mathrm{III}^{\mathrm{c}} \mathrm{s}$. et il est probable que cette installation vinicole a connu d'autres transformations qui seraient marquées par la construction, à un niveau supérieur, des cuves B5 et B11. Un seul point certain : vers 270 après J.-C., on n'a pas hésité à défoncer le béton de la salle S3 pour enfouir un trésor monétaire. Il est probable qu'à partir de cette date, le chai fut abandonné car, au début du siècle suivant, il servit de pièce d'habitation, le bassin B9 étant transformé en dépotoir et le sol étant recouvert d'une couche d'occupation. Ce niveau contenait des déchets alimentaires (huîtres, ossements), des poteries et des monnaies de Constantin et de Constantin II. La production de vin qui aurait commencé dans la seconde moitié du $\mathrm{I}^{\mathrm{er}} \mathrm{s}$. après J.-C. aurait donc duré jusqu'au troisième quart $\mathrm{du} \operatorname{III}^{\mathrm{e}} \mathrm{s}$.

J.-P. B.

\section{SAINTE-Colombe (GIRONDE)}

La villa romaine du bourg de Sainte-Colombe est édifiée à l'extrémité d'un plateau dominant la rive droite de la Dordogne. Le site, connu depuis la fin du XIX ${ }^{c} s$. par la découverte de mosaïques sur le parvis de l'église, a fait l'objet d'une fouille partielle par R. Coste entre 1963 et 1965, à l'est de l'église (Coste, 1969) et de sondages par S. Faravel en 1986 (Faravel, 1989), puis par W. Migeon en 1995 (Migeon, 1996).

La chronologie des bâtiments reste incertaine. Il semble que la villa ait été édifiée à l'emplacement d'un établissement rural de l'âge du Fer, mais on ne sait si sa fondation remonte au Haut-Empire. Les seules données chronologiques disponibles concernent l'Antiquité tardive $\left(\mathrm{IV}^{\mathrm{e}}-\mathrm{V}^{\mathrm{e}} \mathrm{s}\right.$.). La villa comportait alors une pars urbana (située sous l'église et ses abords). Les pièces d'habitation étaient pavées soit de sols en opus signinum, soit de mosaïques à décor géométrique (galerie à l'ouest de l'église) bien caractéristiques de la production des mosaïstes aquitains à la fin de l'Antiquité. Un long mur est-ouest semble relier cette partie résidentielle à un grand bâtiment fouillé par R. Coste entre 1963 et 1965. Cet édifice, dont l'extrémité occidentale est située à $5 \mathrm{~m}$ à l'est du chevet de l'église, n'a pas été intégralement fouillé : seuls les murs périmétraux et un ensemble de bassins ont été dégagés. Le mobilier trouvé lors de ces recherches comprend de la céramique sigillée, des monnaies de la première moitié du $\mathrm{IV}^{\mathrm{e}} \mathrm{s}$. et un vase mérovingien (Gallia, XXIII, 2, 1965, p. 421-422). Mais les conditions de découvertes ne sont pas précisées. La sigillée provient-elle de remblais de construction, les monnaies de sols d'utilisation et le vase mérovingien d'une réoccupation des ruines? La construction et le fonctionnement de l'installation seraient alors contem- 
Fig. 75 - Plan d'interprétation du bâtiment vinicole de la villa du bourg de Sainte-Colombe, Gironde (dessin H. Gaillard, 1999, d'après Coste, 1969).

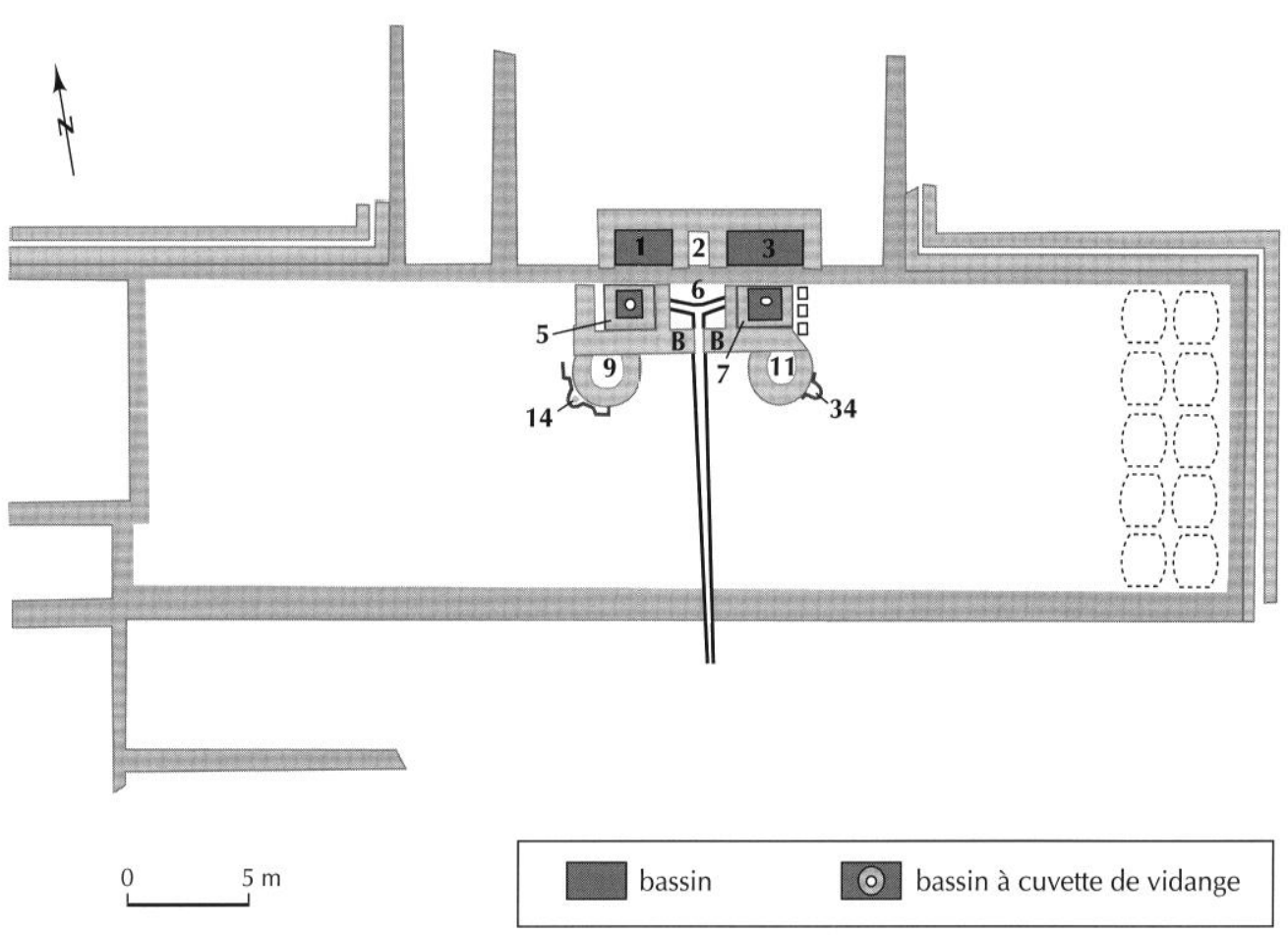

porains de la villa de l'Antiquité tardive ${ }^{174}$. Il semble toutefois acquis que la villa cessa d'être occupée avant les $\mathrm{VI}^{\mathrm{e}}-\mathrm{VII}^{\mathrm{e}} \mathrm{s}$., car ses ruines furent utilisées comme cimetière à partir de cette époque. Les recherches ponctuelles effectuées depuis 1986 ont en effet permis de préciser ce point et de repérer, au nord-ouest de l'église, le fond d'un bassin doté d'une marche et présentant d'épais dépôts de calcite. Il pourrait s'agir d'un bassin régulateur à l'arrivée d'un aqueduc.

Le bâtiment rectangulaire oblong mesure $50 \mathrm{~m}$ de longueur sur $14 \mathrm{~m}$ de largeur (fig. 75). Au nord, on décèle l'existence d'une cour limitée à l'est par un mur et à l'ouest par une longue salle (galerie ?). Au milieu du côté nord du grand bâtiment se trouve un complexe construit à cheval sur le mur de façade. Deux groupes de bassins étagés sont symétriquement aménagés de part et. d'autre de deux pièces (2 et 6 ). Ces pièces percées d'ouvertures axiales servaient de couloir mettant en communication la cour située au nord avec l'intérieur du

174. Mais on ne peut exclure l'hypothèse que la sigillée corresponde à la phase d'utilisation, les monnaies à l'abandon et au comblement des cuves, ce qui entrainerait une attribution de ces structures au HautEmpire : R. Coste (1969, p. 118) signale avoir trouvé un lot de quatre pièces de bronze du IVe $\mathrm{s}$. sur le sol de la salle 6 ; on doit plutôt les attribuer à la phase précédant l'abandon de l'installation qu'à celle de son utilisation optimale car cuves et sols étaient nettoyés chaque année. bâtiment. Par l'intermédiaire des pièces 2 et 6 , on accédait aux bassins 1 et 3 et aux cuves 5 et 7 .

Les deux bassins symétriques 1 et 3 édifiés en saillie mesurent 3,30 m de longueur sur 1,20 m de largeur. Leur sol et leurs parois devaient être en terre cuite ${ }^{175}$. Leur mode de construction et leur disposition permettent d'interpréter ces deux pièces comme des fouloirs à vin protégés par un auvent. Le moût devait s'écouler dans les deux cuves 5 et 7 placées en contrebas, de l'autre côté du mur de façade, à l'intérieur de la salle. Les cuves mesurent $1,25 \mathrm{~m}$ par $1,10 \mathrm{~m}$ environ pour une profondeur utile de $0,75 \mathrm{~m}$ environ. Chacune d'elles était donc susceptible de recueillir $10 \mathrm{hl}$ de moût. Comme les fouloirs 1 et 3 , le sol et les parois des deux cuves étaient pavés de carreaux de dimensions variables (de $30 \mathrm{~cm} \times 40 \mathrm{~cm}$ pour la cuve 5 , de $55 \mathrm{~cm} \times 60 \mathrm{~cm}$ pour la cuve 7). Toutes deux possédaient une cuvette hémisphérique de vidange en leur centre, celle du bassin 5 ayant été creusée dans un bloc de pierre et celle du bassin 7 ayant probablement fait l'objet d'une réfection.

Au sud des cuves, les pièces 9 et 11 , très arasées lors de la fouille, présentent des dispositions symétriques

175. Tous les carreaux avaient disparu à l'exception d'un fragment sur l'une des parois (information aimablement communiquée par R. Coste). 
inédites. Il s'agit d'espaces en forme de demi-cercle outrepassé d'un diamètre de $2,70 \mathrm{~m}$, ceinturés par un mur large de $0,57 \mathrm{~m}$. Ces structures qui étaient abritées par la toiture du bâtiment rectangulaire doivent correspondre à des socles. Selon R. Coste, "le remplissage entre les murs est un parquet de béton à gros fragments de tuileau ». Il est vraisemblable que la surface du sol n'était pas conservée et que ce béton correspondait à une couche intermédiaire, un rudus, qui devait être recouvert d'autres couches de béton de pierres et de béton de tuilcau. Contre les flancs est et ouest des pièces 9 et 11 se trouvent deux ensembles de canaux et deux petites cuves 14 et 34 . En forme de demi-cylindre de $0,67 \mathrm{~m}$ de diamètre, enduites de béton de tuileau, ces dernières comportaient une cuvette de vidange de $0,25 \mathrm{~m}$ de diamètre. Leur profondeur, dépendant de l'épaisseur des socles 9 et 11, n'est pas connue. Mais si l'on restitue le sol de 9 et 11 au niveau de celui de la salle 6 , la profondeur des cuves pourrait avoir dépassé $0,50 \mathrm{~m}$. Leur contenance minimale était donc de l'ordre d'une centaine de litres.

Le mode de construction du sol des pièces 9 et 11 évoque celui d'une dalle de pressoir. Les capacités des cuves 14 et 34 , nettement moindres que celles des cuves placées sous les fouloirs, conviendraient bien au recueil du jus de presse, moins abondant que le jus de goutte. Outre ces observations, la position des deux ensembles 9-14 et 11-34 dans la chaîne opératoire rend vraisemblable leur identification comme pressoirs. La forme quasi circulaire des socles maçonnés 9 et 11 indique peutêtre qu'il s'agit de presses à vis directe, plutôt que de presses à levier.

Le jus de goutte et de presse devait être périodiquement transvasé dans des récipients où s'opérait la fermentation. Nous émettons l'hypothèse qu'il s'agit de foudres qui étaient logés dans le grand bâtiment long de $50 \mathrm{~m}$.

J.-P. B. et C. B.

\section{VITICULTURE ET VINIFICATION EN AQUITAINE ROMAINE}

L'ensemble des données recueillies renouvelle singulièrement notre appréciation sur la viticulture dans l'Aquitaine gallo-romaine et permet d'apporter un certain nombre de remarques synthétiques sur divers aspects de la production du vin ayant trait à la technique (cépages, outils, vinification) et à la chronologie. Ce dernier point, toutefois, comporte encore bien des zones d'ombre, en l'absence de données archéologiques précises pour nombre d'installations. Aussi faut-il souhaiter que ce programme collectif de recherche suscite de nouvelles recherches archéologiques sur les installations vinicoles de ces régions de la Gaule du Sud-Ouest.

\section{LES CÉPAGES}

Columelle (De l'agricullure, III, 2, 19) et Pline l'Ancien (H. N., XIV, 26-27) nous font savoir que dès le I Ir s. la Vitis Bilurica ou Bilurigiaca était un cépage d'un certain renom, et selon toute vraisemblance il s'agit d'un plant cultivé chez. les Bituriges Vivisques (voir supra). Il semblerait ainsi que dès la seconde moitié du I ${ }^{\text {er }} \mathrm{s}$. après J.-C. le vignoble bordelais se soit taillé une place de choix dans l'agriculture et l'économie romaines.

Sur le terrain, pour ces périodes, les traces archéologiques sont plus rares. Les fouilles de l'îlot SaintChristoly à Bordeaux en 1982 ont bien livré des multitudes de grappes de raisins et de pépins dans le fond d'un puits d'un bâtiment public (marché ?), mais il fut difficile d'affirmer avec certitude la provenance locale de ces produits (Debord, Gauthier, 1982, p. 56-60). Ces pépins furent soumis en novembre 1982 pour étude à J.-P. Doazan, ingénieur à l'INRA (Unité de recherches sur les espèces fruitières et la vigne). Dans un courrier conservé dans le rapport de fouilles, il écrit : « Le pépin est généralement considéré par les ampélographes comme un organe très utile pour la distinction des espèces; par contre, il ne suffit pas à caractériser les variétés (ou cépages) composant l'espèce Vitis vinifera $\mathrm{L}$. Nous avons fort heureusement constitué une petite collection de pépins d'un certain nombre d'espèces et variétés auxquels nous avons pu comparer le lot provenant de Saint-Christoly. Je crois pouvoir affirmer qu'il s'agit de pépins de l'espèce européenne Vitis vinifera $\mathrm{L}$., ce qui ne surprendra guère. De plus, ils semblent nettement différents des quelques lots de vignes sauvages que nous détenons : ces derniers présentent très généralement un bec beaucoup plus court. Enfin, si l'on se réfère aux cépages actuellement existants dans notre région de Bordeaux (rouges: cabernet franc, cabernet-sauvignon, fer-servadou, merlot, petit verdot; blancs: muscadelle, sauvignon, 
sémillon, ugni blanc), celui dont les pépins ressemblent globalement le plus est le petit verdot. En conclusion, cet examen superficiel et rapide laisse supposer qu'il s'agit de pépins de vigne cultivée. Mais tout rapprochement avec des cépages actuels ne peut que relever de la pure hypothèse."

Toujours à Bordeaux, à quelques mètres du même bâtiment public, en bordure de la Devèze, ont été découverts, fait rarissime en Occident, des ceps de vigne (cinq) portant des traces de taille; ils avaient été écrasés par un énorme remblai venu les recouvrir dans la seconde moitié du I ${ }^{\mathrm{er}} \mathrm{s}$., lors des réaménagements des berges. Un seul pied put être dégagé convenablement et il est aujourd'hui présenté au Musée d'Aquitaine. Un quinaire d'argent d'Auguste fut trouvé à l'occasion de cette fouille d'urgence associé au pied de vigne ${ }^{17 \hbar}$. Mais ces vignes taillées sous le règne de Tibère furent-elles utilisées pour produire du vin ou du raisin de table?

Pour l'Antiquité tardive, on possède maints témoignages littéraires concernant les vignobles du Bordelais. En revanche, les traces archéologiques sont quasiment inexistantes, faute peut-être d'avoir pu identifier avec certitude les structures de production dans les nombreuses villae explorées en Aquitaine. À Bordeaux même, la seule mention archéologique de la présence de vigne pour cette période est apportée par les récentes analyses palynologiques effectuées lors de la fouille de la Cité judiciaire de Bordeaux ${ }^{177}$ qui ont permis d'identifier, au fond d'un fossé de drainage daté du $I V^{\mathrm{e}}-\mathrm{V}^{\mathrm{e}} \mathrm{s}$., des pollens de vigne (Vitis) associés à du génévrier (Juniperus) et du noyer (Juglans).

On s'est enfin tourné vers les représentations de vigne sur les mosaiqques et les bas-reliefs de l'Antiquité tardive de ces régions méridionales de l'Aquitaine. Mais ces images, pour aussi belles et précises qu'elles soient, ne permettent guère d'identification variétale. Les ampélographes bordelais consultés (P. Leclair et J.-P. Doazan, INRA) considèrent les représentations comme trop "figuratives " et " pas assez fidèles, botaniquement parlant ». Seule la mosaique de la villa du Palat à SaintÉmilion, à petite feuille trilobée et à grappe à petit

176. P. Debord, M. Gauthier, Rapports de fouilles de l'îlot SaintChristoly, 1982, archives du Service régional de l'archéologie d'Aquitaine, 1 vol.

177. Cf. Sireix dir., 1996, vol. 9, p. 52 et 56 ; analyses palynologiques réalisćes par M.-F. Diot du Centre national de Préhistoire.

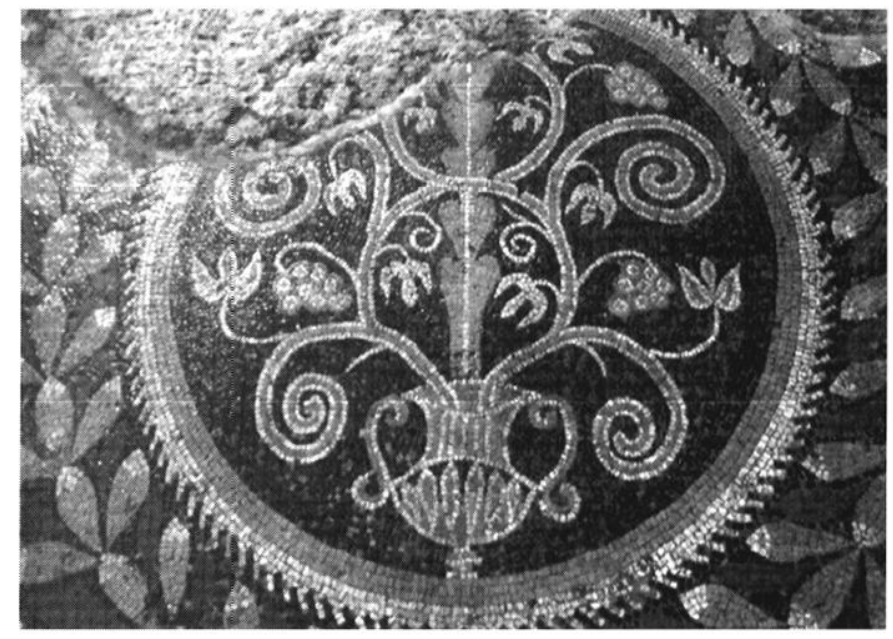

Fig. 76 - Mosä̈que à décor de vigne de la villa du Palat à SaintEmilion, Gironde. Le rendu de la feuille el de la grappe fait penser à la Vitis vinifera dite lambrusque.

nombre de baies (fig. 76), pourrait évoquer une forme sauvage de la Vitis vinifera $\mathrm{I}$. dite lambrusque et qui subsiste encore dans un petit nombre de sites européens (J.-P. Doazan).

D. B. et P. R.

\section{LES OUTILS DE VIGNERONS DE L'AQUITAINE ANTIQUE}

Parallèlement aux recherches menées sur les vestiges bâtis des établissements vinicoles du Grand Sud-Ouest, nous avons rassemblé les informations disponibles sur les outils de vignerons (fig. 77). À partir des exemples mis au jour en Saintonge, nous proposons ici une synthèse qui devra être peaufinée par la suite lorsque les données de terrain seront plus importantes.

Il semble qu'il faille distinguer trois types d'outils de vignerons, chacun correspondant à une tâche spécifique d'entretien du vignoble : la "serpe à talon », la " serpe de vigneron " et la « serpette " à vendanger (fig. 78).

En période hivernale, lorsque la végétation se repose, le viticulteur entame une phase déterminante de son activité : la taille. Il doit rendre un diagnostic rapide de l'état sanitaire de chaque cep, éliminer les parties du souchot qui sont mortes ou dont la vigueur est moindre, couper les sarments pour garder uniquement quelques brins parmi les plus vivaces qui feront la récolte prochaine. Pour ce faire, on utilisait la "serpe à talon » 


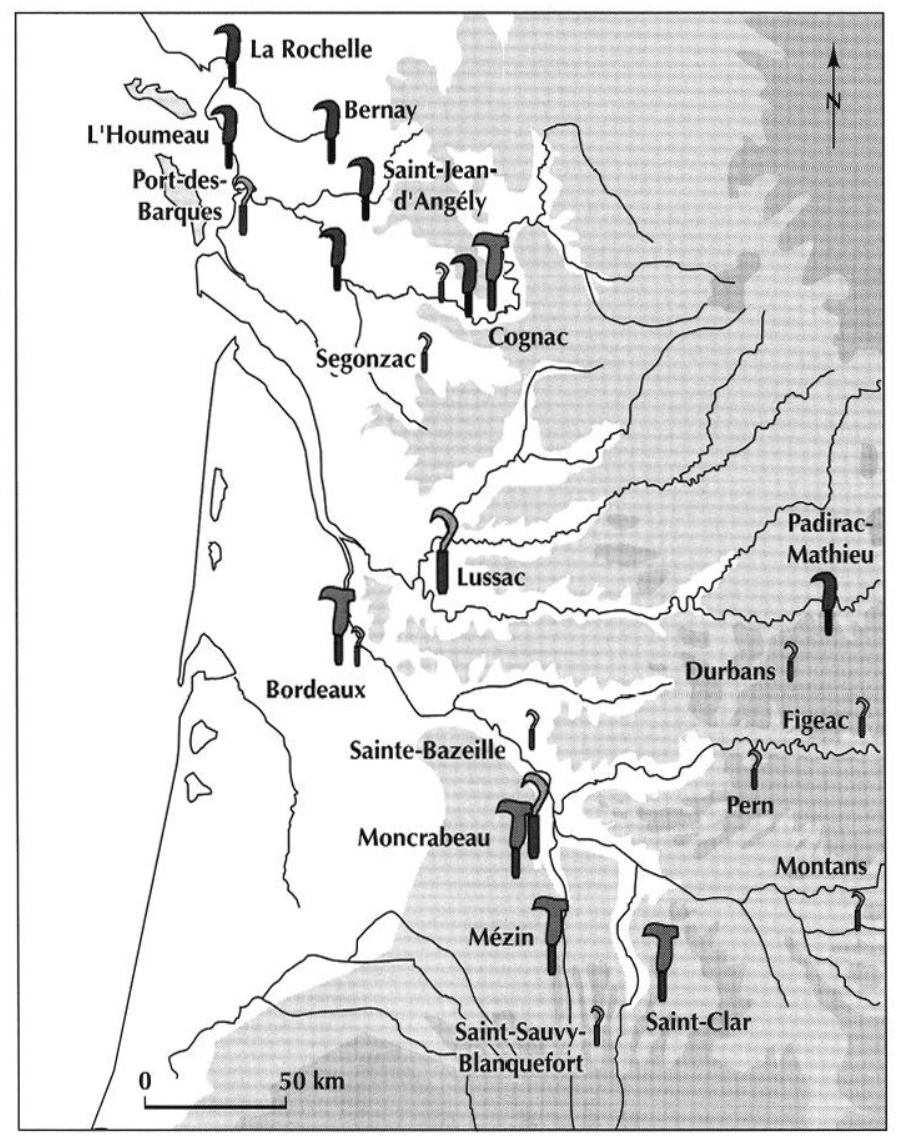

37 serpettes à vendanger $(1,2,5)$

Fig. 77 - Carte de répartition des serpes et serpettes d'après les travaux menés dans le cadre du programme collectif de recherche (dessin H. Gaillard, 1999).

ou " serpe à ergot ». Son appendice tranchant, rapporté sur le dos de la lame, facilitait cette action de taille par coups répétés.

C'est vraisemblablement ce que les auteurs antiques nommaient la falx vinitoria (Saint-Denis, 1953). Columelle en fait une description détaillée en nommant chacune des parties (IV, 25). C'était un outil polyvalent qui permettait également la taille des sarments, grâce au tranchant courbe cette fois. En Aquitaine peu d'exemplaires sont connus. Dans la villa de Bapteste, près de Moncrabeau (voir supra, p. 136-138), il s'agit d'une serpe en fer, à emmanchement à soie, dont la longueur est de $22 \mathrm{~cm}$ pour une largeur du tranchant de $3,8 \mathrm{~cm}$ (fig. $78, \mathrm{n}^{\circ} 1$ ). Un autre exemplaire est signalé dans le
Lectourois (Barbé, 1984). Il est vraisemblable qu'une lame de fer découverte à Cognac corresponde à un talon de serpe (Vernou et al., 1990, p. 76, n 212). Enfin un bel exemplaire à douille a été recueilli récemment à Portdes-Barques (information P. Duprat).

$\mathrm{La}$ « serpe de vigneron » est voisine de la précédente mais elle n'a pas de talon et les courbes de l'outil sont plus marquées : dos de l'outil en arrière de l'axe d'emmanchement, profil quasi sinusoïdal du tranchant. Ce type de serpe, particulièrement léger et maniable, était idéal pour la taille des sarments. De nombreux exemplaires sont connus en Saintonge. À Cognac, sur le site de La Haute-Sarrazine (Vernou et al., 1990, p. 76, $\mathrm{n}^{\circ} 210$ ), l'une d'elles avait un emmanchement à douille et une longueur de $22 \mathrm{~cm}$ (fig. $78, \mathrm{n}^{\circ} 2$ ). D'autres sont signalées en Aunis, à La Rochelle sur le site de La Pointe des Minimes (Flouret et al., 1984, p. 22) ou à L'Houmeau sur le site du Haut-Pampin (Laporte, Texier, 1980, p. 117).

Pour le dernier type et uniquement pour celui-là, on doit réserver la dénomination "serpette ». L'outil est adapté à l'action de taille de la queue du raisin par un mouvement de rotation tout en prenant la grappe de l'autre main. La "serpette" est de taille modeste (12 à $15 \mathrm{~cm}$ de longueur). Son emmanchement est assuré par une soie, dont l'extrémité est parfois retournée à la base. du manche. La lame courbe forme un petit croissant. On la nomme différemment d'une région à l'autre; en Charente on emploie le terme de "guignette ». Dans l'Antiquité, Caton parlait de falcula. Plusieurs exemplaires ont été découverts dans l'Aquitaine antique: à Cognac encore (Vernou et al., 1990, p. 76, n²13) où la serpette mesure seulement $12 \mathrm{~cm}$ (fig. 78, $\mathrm{n}^{\circ} 3$ ), à Segonzac, sur les terres de Fontbelle (Vernou, Baigl, 1991, p. 106) dont la soie de l'outil est torsadée, à Portdes-Barques (information P. Duprat), à Lussac dans la villa de Barat où les cinq serpettes mesuraient entre 13 et $15 \mathrm{~cm}$ (Garde, 1959, p. 8). Les fouilles récentes de la Cité judiciaire à Bordeaux ont révélé un magnifique exemplaire (inédit, information C. Sireix) qui confirme l'activité viticole de Burdigala où un autre fragment avait été découvert sous la place des Grands-Hommes (Feugère, 1997, n 63).

Ce type d'instrument est très ancien et a perduré jusqu'à une époque récente de l'histoire de la viticulture. Il est mentionné dans l'Encyclopédie de Diderot et d'Alembert (Agriculture, Culture de la vigne, pl. I 

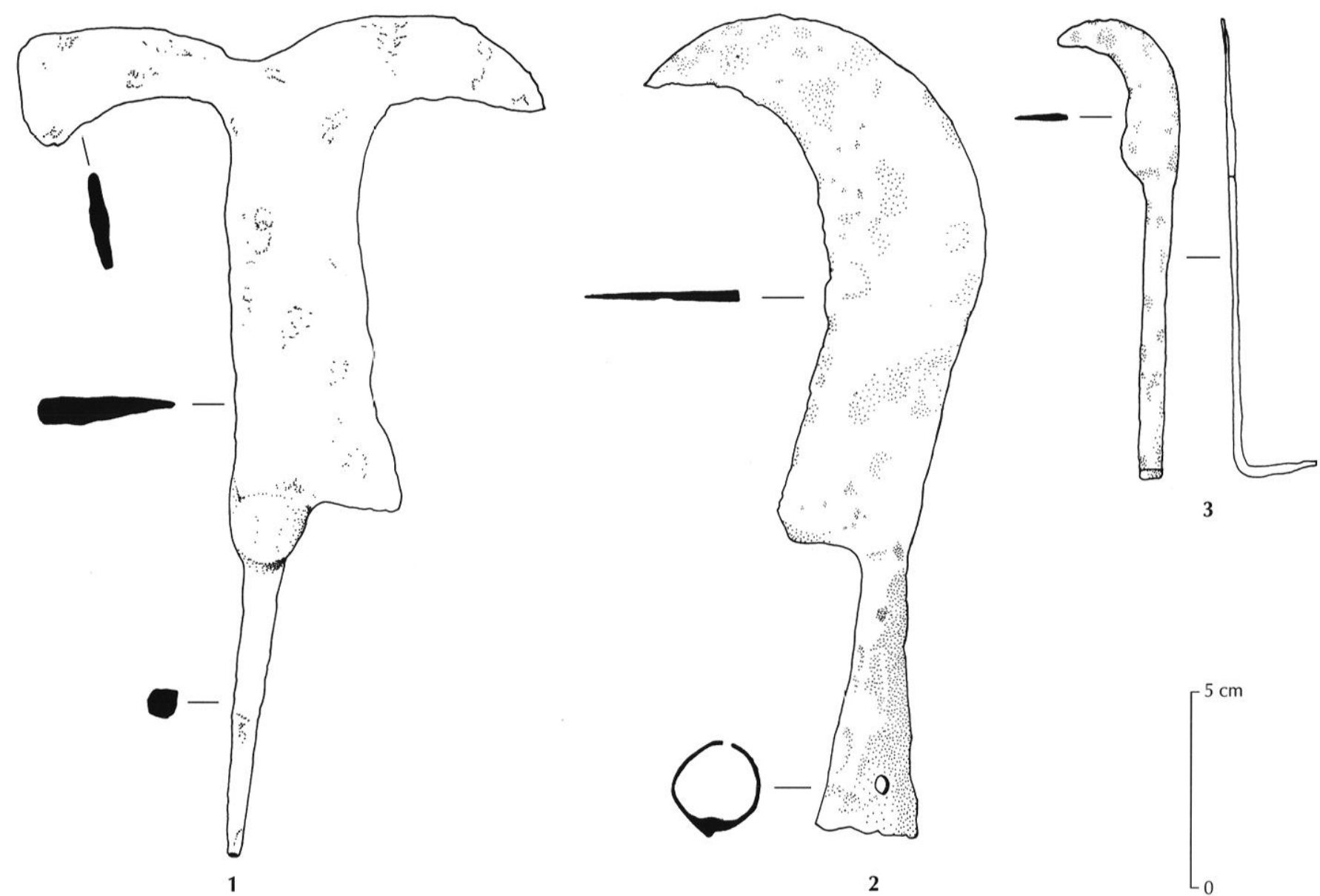

3

Fig. 78 - Outils de vignerons : 1, serpe à talon (falx vinitoria), villa de Bapteste à Moncrabeau, musée de Nérac; 2, serpe de vigneron, villa de La Haute-Sarrazine, musée de Cognac; 3, serpette à vendanger (falcula), villa de La Haute-Sarrazine, musée de Cognac (dessin C. Vernou-Magister).

et II). Les musées ethnologiques conservent encore de nombreux exemplaires de ces " couteaux à vendanger " qui ont précédé l'invention du sécateur métallique (Garde, 1959, p. 9). Ce dernier s'est imposé progressivement au cours du Second Empire, mais certains auteurs pensent que les «forces » antiques (forfex) ont pu servir à la cueillette des raisins. Cela paraît vraisemblable pour la cachette d'outillage d'un paysan viticulteur de Tarquimpol, en Moselle (Ferdière, 1988, p. 92). Signalons pour information qu'un outil de ce genre a été mis au jour sur le site cognaçais de La Haute-Sarrazine (Vernou et al., 1990, p. 78, ${ }^{\circ} 218$ ).

Ces premiers éléments encouragent à mener une enquête plus large, à l'échelle du Grand Sud-Ouest pour le moins.

C. V.

\section{TECHNIQUES AGRICOLES ET VINIFICATION}

La base documentaire établie par le programme collectif de recherche comprend 91 installations attribuables à la production du vin en Aquitaine, mais seulement une dizaine d'entre elles sont suffisamment préservées pour permettre une interprétation. Toutefois, le recoupement des observations et les comparaisons avec les installations méditerranéennes autorisent une restitution des procédés les plus couramment employés.

\section{LES TRACES DE CULTURE}

La fouille de Reignac a probablement mis au jour, outre une partie d'installation vinicole, les vestiges de 


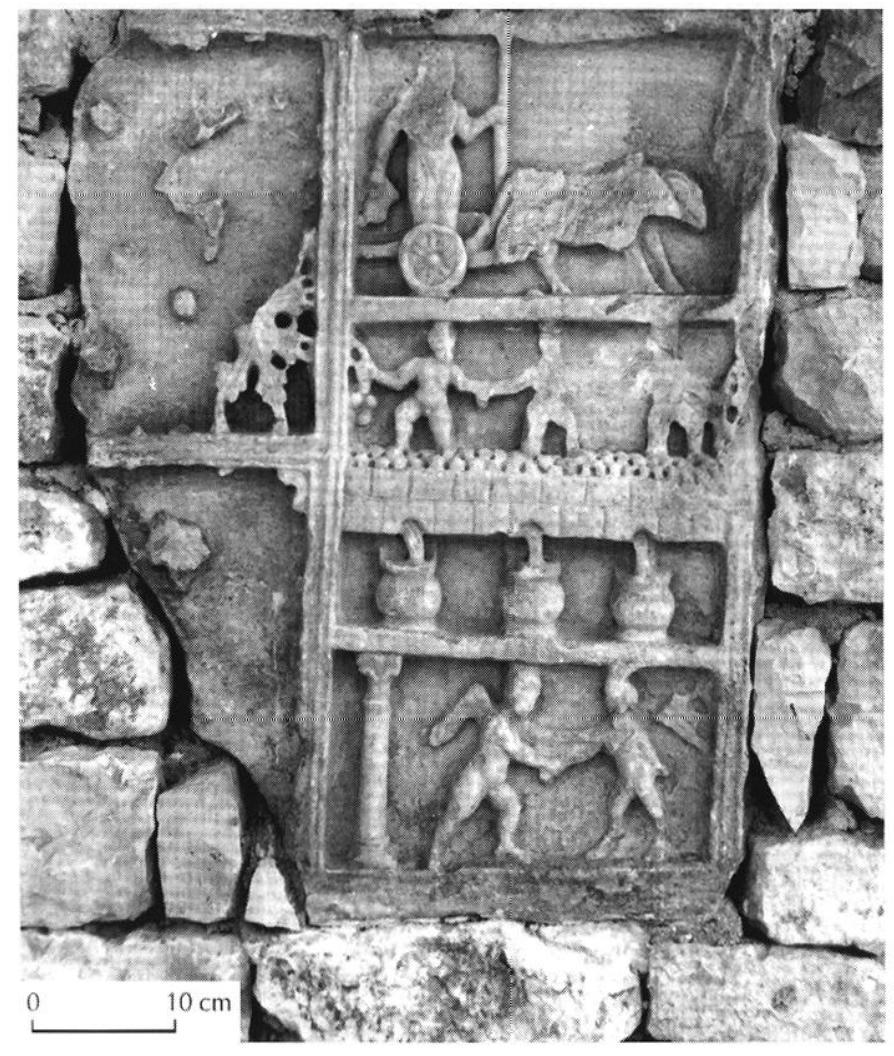

Fig. 79 - Sarcophage de Cahors, à scène de foulage (cliché D. Rigal).

deux champs de vignes caractérisés par des fosses de plantation allongées convenant pour deux pieds de vignes (fig. 70). Régulièrement implantées, elles permettaient une circulation aisée entre les ceps et un ensoleillement maximal. Les traces relevées peuvent être comparées à celles récemment décelées à Lapalud dans le Vaucluse qui ont, elles aussi, la forme de rectangles (voir supra, fig. 20, p. 61).

\section{LES FOULOIRS}

En début de chaine opératoire, on trouve systématiquement le fouloir, une cuve dans laquelle les hommes écrasaient les raisins avec les pieds (fig. 79). Cette pièce était pourvue d'une évacuation vers unc cuve située en contrebas. L'arasement des vestiges a généralement entraîné la destruction du fouloir et scule subsiste la cuve de recueil du moût. Les sites d'Allas-les-Mines (fig. 62), de Montcaret, de Cadillac (fig. 80), de Sainte-Colombe (fig. 75) et de La Haute-Sarrazine à Cognac (fig. 71) ont conservé tout ou partie du sol de leurs fouloirs. Il s'agit
Fragment de la face antérieure d'un sarcophage en marbre blanc dont la composition d'ensemble est connue par un dessin médiocre $d u X V I I I^{e} s$. L'un des petits côtés présente l'image d'Adam el Ève.

Dimensions de la cuve (d'après la description de P.-L. de Besombes de Saint-Geniès) : long. : 2,16 m, larg. : 0,71 m, haut. : 0,44 m.

Le sarcophage était encore visible au XVIII s. dans le cloître de la cathédrale de Cahors. Il n'en subsiste aujourd'hui que des fragments, dont celui-ci qui a été retrouvé récemment, encastré dans une construction moderne; d'autres sont conservés au musée de Cahors. Datation : seconde moitié du VT s. d'après Briesenick, mais sans doute plus tôt.

On voit ici la partie centrale de la face antérieure du sarcophage qui est subdivisée en trois registres superposés, tous décorés de sujets dionysiaques.

- Au registre supérieur, Dionysos debout sur un char attelé par deux béliers (boucs?); le dieu tient un canthare de la main droite et s'appuie de la main gauche sur un thyrse.

- Au registre médian, trois Amours se tenanl par la main foulent le raisin; le moût s'écoule dans trois dolia.

- Au registre inférieur, la lutte d'Éros et de Pan.

Il a semblé intéressant de publier cette photographie, mais il ne faut pas manquer de rappeler que la scène de foulage est souvent associée aux sarcophages romains à représentations dionysiaques (voir Turcan, 1966).

BIBLIOGRAPHIE : Fontenilles, 1879, dessin entre p. 576 et 577 ; Le Blant, 1886, n० 87, p. 70-71; Briesenick, 1962, n 17, pl. 16, 5 (fragment conservé au musée de Cahors).

d'une pièce rectangulaire, mesurant $2,30 \mathrm{~m}$ sur $1,40 \mathrm{~m}$ à Cadillac, de $4 \mathrm{~m}$ à $6,60 \mathrm{~m}$ de longueur et $1,20 \mathrm{~m}$ de largeur à Allas-les-Mines, 3,30 m sur 1,20 m à SainteColombe, $9 \mathrm{~m}$ de longueur pour une largeur de $5 \mathrm{~m}$ au moins à La Haute-Sarrazine. À Mérignas, bien que le sol ait disparu, on reconnait le fouloir mesurant $5,50 \mathrm{~m}$ sur 2,50 m (fig. 81) ${ }^{178}$. La fondation du sol est empicrrée et, dans une première phase, il est associé à deux cuves; il est possible que, outre la fonction de fouloir, la pièce ait servi de pressoir.

Les fouloirs et les cuves sûrement datables du HautEmpire, comme ceux de Ia Haute-Sarrazine, d'Allas-lesMines ou de La Pointe des Minimes sont intérieurement enduits de bćton de tuileau. Mais il existe une autre série d'installations, peut-être plus tardives, dont les fouloirs et les cuves sont pavés de dalles de calcaire tendre ou de briques : Sainte-Colombe, Cadillac et Montcaret.

178. Cf. Sion, 1994, p. 306. Le plan, jusqu'à présent inédit, est conservé dans les archives du Service régional de l'archéologic d'Aquitaine. 


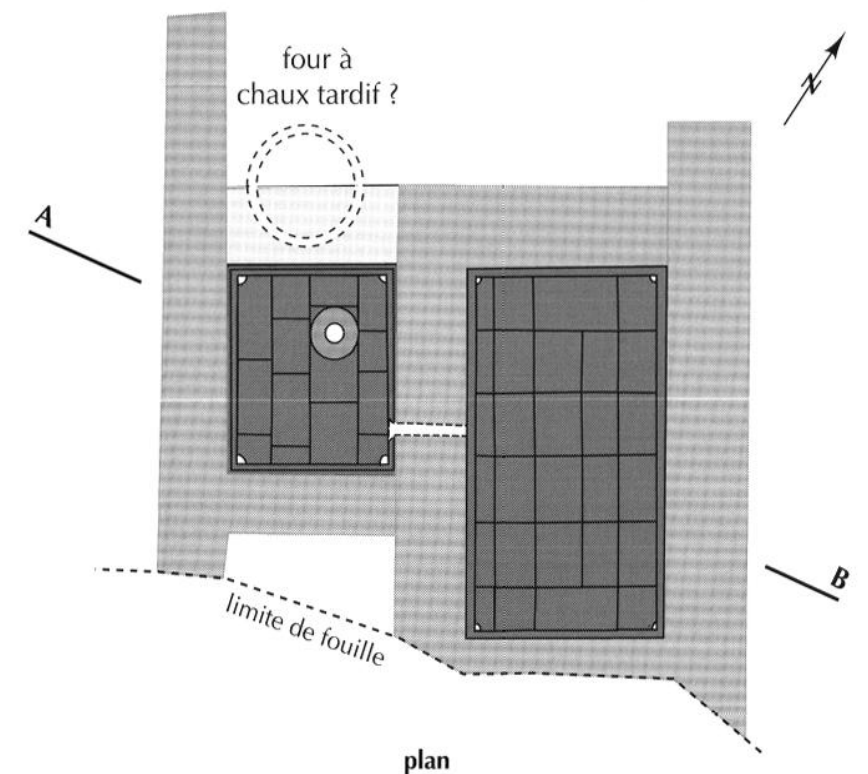

A

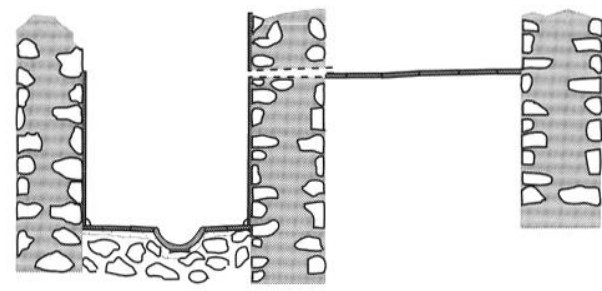

coupe

bassin

(2) bassin à cuvette de vidange

Fig. 80 - Plan et coupe du fouloir et de la cuve de recueil du mô̂t à cuvelle de vidange de Cadillac, Gironde (dessin H. Gaillard, 1997, d'après le plan de A. Pezat, 1955, cf. Coupry, 1957, p. 248, fig. 10).

\section{LES CUVES DE RECUEIL DU MOÛT}

Du fait de leur préservation en sous-sol, ce sont les cuves qui signalent l'existence des installations viticoles (fig. 61). Leurs dimensions varient de $1,25 \mathrm{~m} \mathrm{x} 1,10 \mathrm{~m}$ à Sainte-Colombe à 5,50 m x 1,70 m x 2,05 m à La HauteSarrazine (bassins jumelés 10 et 11 d'une contenance unitaire de $191 \mathrm{hl}$ ). Aucun groupement fondé sur la taille des cuves ne se dessine. Les cuves de La Haute-Sarrazine, comme l'ensemble de l'installation, se distinguent par leur nombre et leur grande capacité. Il eût été intéres- sant de comparer leurs dimensions avec celles des cuves de La Pointe des Minimes (La Rochelle), mais la publication de ces dernières ne donne pas toutes les données métriques.

Une majorité de cuves, creusées dans le substrat, sont ceinturées par quatre murs recouverts d'un enduit de béton de tuileau (par exemple à La Haute-Sarrazine, à Chives, à La Rochelle, etc.). Les fonds sont formés d'une dalle de béton de tuileau coulée sur un hérisson de pierres, l'étanchéité entre les parois et le fond étant assurée par des joints en quart-de-rond. Le fond de la cuve comporte toujours une cuvette de vidange en forme de portion de sphère ménagée au centre ou dans un angle de la cuve. Son diamètre, variable, est fréquemment de l'ordre d'un pied. Dans les cuves enduites de mortier de tuileau, la cuvette est en général intégrée au sol; toutefois, celle de Lagorce en Gironde est taillée dans un fond de dolium et à La Haute-Sarrazine l'une d'entre elles est monolithe (fig. 72). Dans les cuves carrelées, cette cuvette est souvent formée d'un bloc de pierre évidé ou réutilise une poterie (à Montcaret en Dordogne et à Vertheuil en Gironde).

Bien que ce détail n'ait pas été systématiquement signalé dans les publications anciennes, bon nombre de ces cuves comportaient un escalier permettant d'accéder aisément au fond. En effet, la plupart des installations n'étaient pas équipées de tuyaux pour transvaser le moût et il fallait donc le puiser avec des seaux. Des escaliers sont signalés à La Rochelle, au Château-d'Oléron, à Nieul-sur-Mer, à Saint-Martial-de-Mirambeau, à Saintes (Les Trains) et à Soubise (Charente-Maritime), à Montcaret et à Saint-Vincent-le-Paluel (Dordogne), à Sainte-Colombe et à Vertheuil (Gironde) et à Nohic (Tarn-et-Garonne).

Les cuves trouvées à Royan (fig. 68), Saint-Georgesd'Oléron et Bords, Saint-Martial-de-Mirambeau, Puyréaux (fig. 84), Montcaret, Cadillac (fig. 80), Cénac, Fronsac, Lugasson, Mérignas (fig. 81), Sainte-Colombe, Vertheuil forment une série présentant la particularité d'être carrelées soit en dalles de calcaire, soit en briques. Dans le chai de Belmont à Royan, K. Robin a observé que les sols carrelés succédaient aux sols bétonnés et pouvaient être datés du IV $\mathrm{V}^{\mathrm{s}}$. À Mérignas (fig. 81), la cuve carrelée a été ajoutée postérieurement à deux cuves primitives enduites de béton de tuileau. Ces indications sont toutefois insuffisantes pour attribuer cette caractéristique technique à 


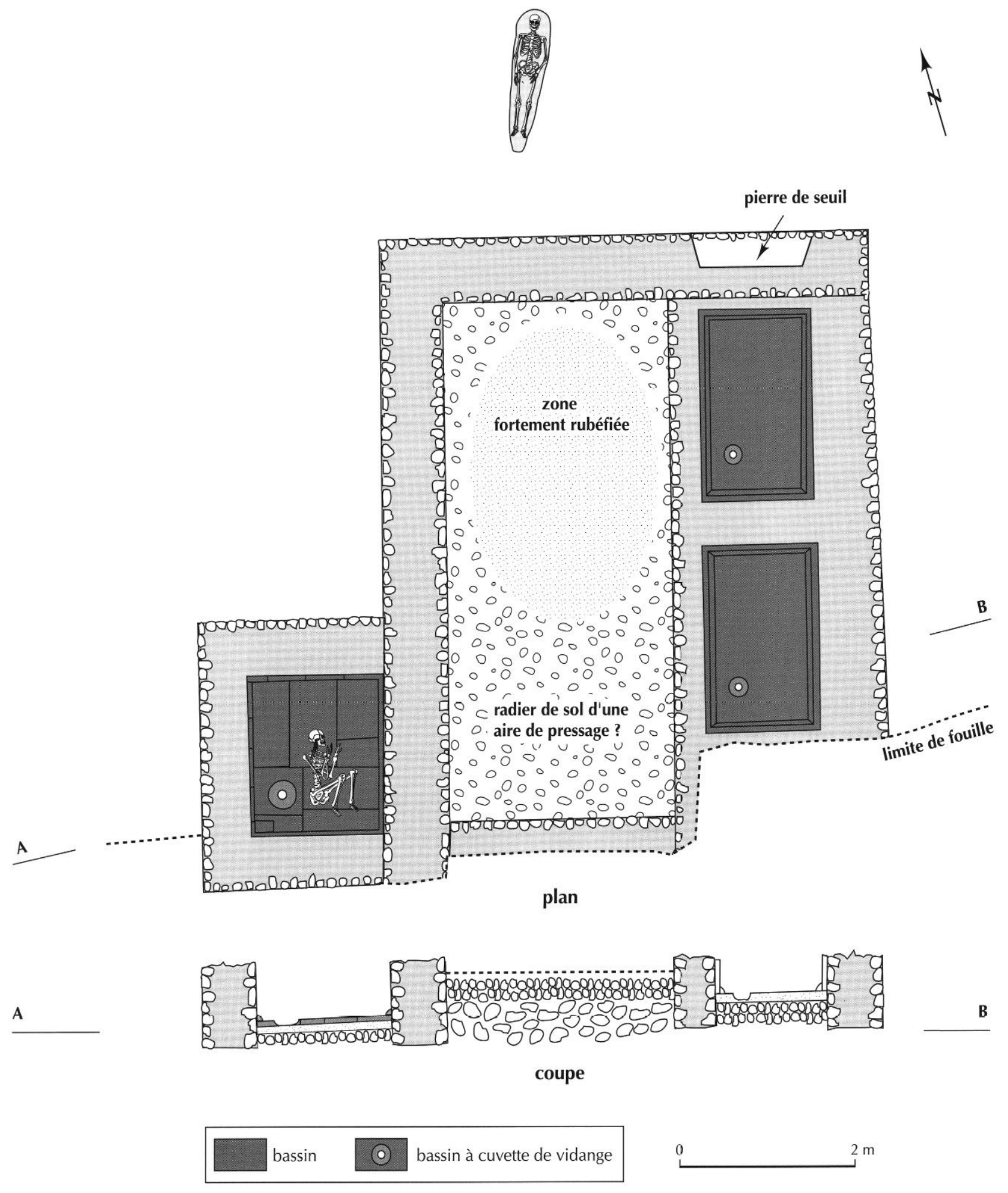

Fig. 81 - Plan et coupe du fouloir (au centre) et des cuves de recueil du moût à cuvette de vidange de Mérignas, Gironde : celle du côté ouest relève d'une seconde phase (dessin H. Gaillard, 1997, d'après le plan de A. Pezat, 1954 conservé dans les archives du SRA d'Aquitaine). 


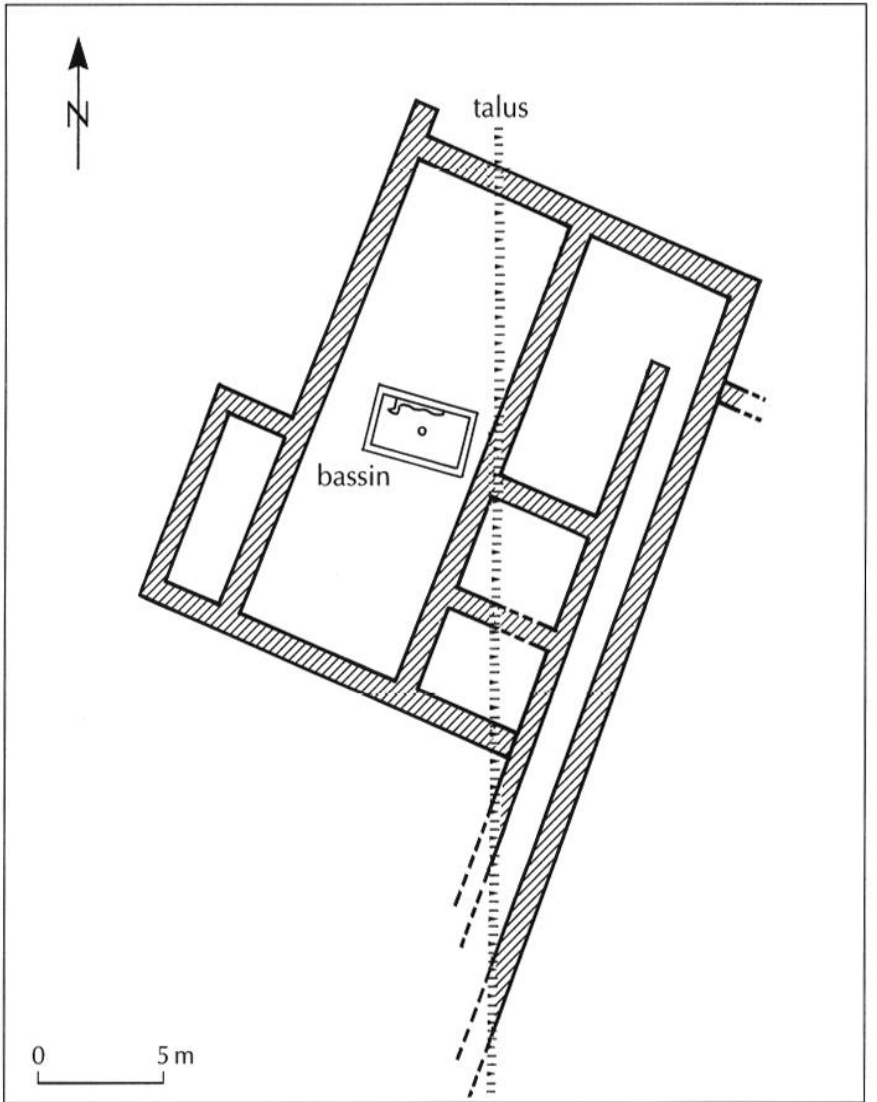

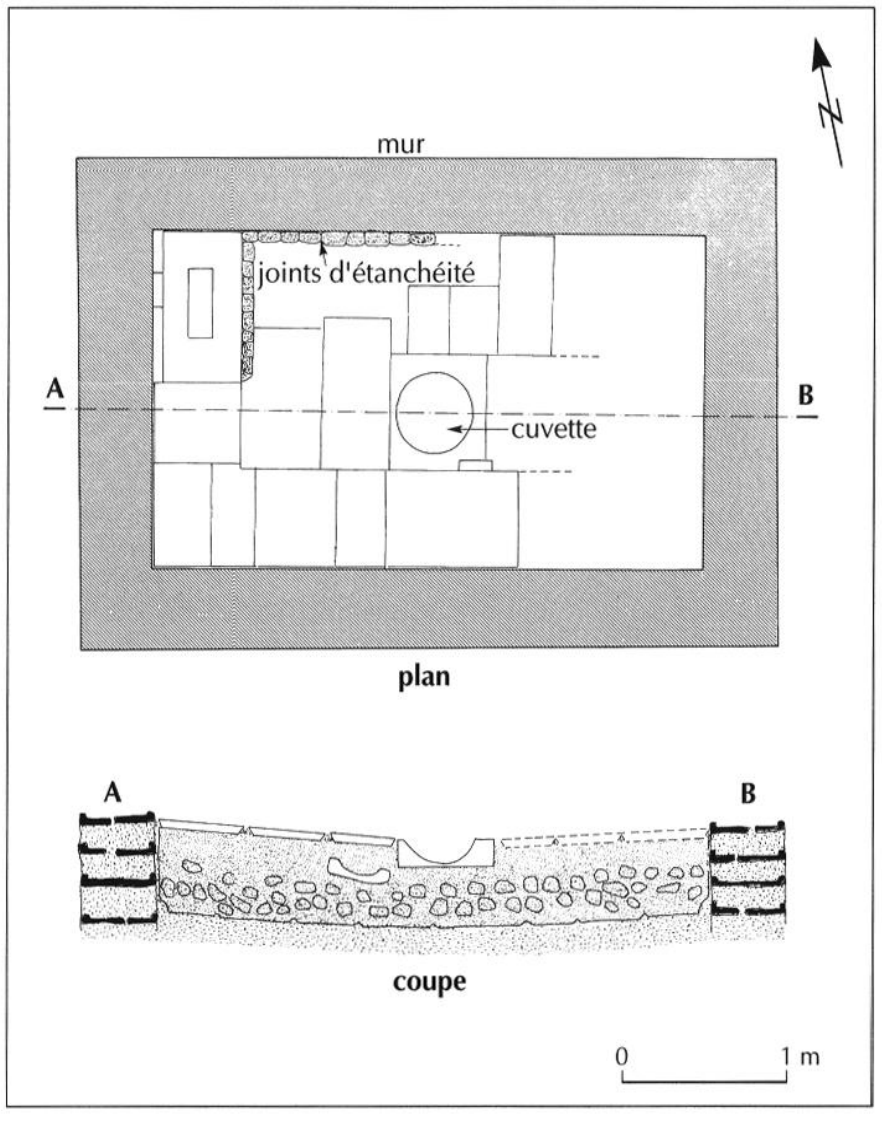

2

Fig. 82 - Cuve de recueil du mô̂t du Chêne à Saint-Martial-de-Mirambeau, Charente-Maritime: 1, plan d'ensemble; 2, plan et coupe du bassin à cuvette de vidange réparée à plusieurs reprises (dessin J. Gaillard, 1979, d'après Maurin, 1999, p. 272, fig. 273a).

l'Antiquité tardive ${ }^{179}$, mais la question doit être posée afin que les recherches futures éclairent ce point.

Malgré le soin mis à assurer l'étanchéité des cuves, il arrivait que l'on constate des fuites du précieux liquide. Il convenait alors de refaire le sol en coulant une nouvelle couche de béton de tuileau ou un nouveau carrelage posé sur du mortier. Ce type de réfection a été constaté à plusieurs reprises, notamment à Royan, où les cuves ont été réparées plusieurs fois et à Saint-Martial-deMirambeau où le carrelage ancien a laissé son empreinte (Maurin, 1999, p. 271-272, n 362) (fig. 82).

179. À Montcaret, la cuve contenait une amphore de Gaza (Berthault, 1998, p. 454), ce qui semblerait indiquer que l'installation a fonctionné jusqu'à l'Antiquité tardive, mais les sites de Saint-Martial-de-Mirambeau en Charente-Maritime, de Fronsac et de Lugasson en Gironde seraient, quant à eux, datables du Haut-Empire.

\section{LES PRESSOIRS}

En Haute-Garonne, un bloc d'assise de pressoir à levier est réemployé dans le chevet de l'église de SaintJean-des-Vignes à Saint-Plancard. Le bloc, en marbre, mesure $1,38 \mathrm{~m}$ de longueur et sa largeur dépassait $0,38 \mathrm{~m}$. Il est creusé à sa face supérieure de deux mortaises rectangulaires qui bloquaient le pied de deux forts montants de bois entre lesquels était fiché le tronc d'arbre servant à écraser le marc. Ce type de pressoir décrit par Caton (Agr., 18) est très fréquent en Méditerranée tant pour l'huile que pour le vin.

À Allas-les-Mines, une aire bétonnée dotée d'une évacuation vers une jarre était située à l'est du fouloir et de sa cuve (fig. 62). L. Maurin l'avait identifiée à raison comme un pressoir à levier dont le praelum devait être encastré dans le gros massif de maçonnerie flanquant le côté ouest de l'aire bétonnée. En revanche, on ne connaît 
pas le mode de manœuvre du pressoir ; étant donné que les fouilles n'ont mis au jour aucune maçonnerie susceptible d'avoir servi d'assise à des montants de treuil, il faut peut-être envisager que le treuil ou la vis était fixé sur un contrepoids de pierre qui aurait été anciennement enlevé.

Les sondages effectués en 1999-2000 par C. PetitAupert et P. Sillières à Lestagnac (Saint-Mézard, Gers) ont mis au jour les vestiges d'un pressoir à levier et vis du Haut-Empire. Dans la villa de Bapteste à Moncrabeau, les fouilles ont dégagé une empreinte (fig. 65) laissée dans l'argile par le socle d'un pressoir à vis directe en bois daté de la première moitié du $\mathrm{II}^{e} \mathrm{~s}$. après $\mathrm{J}$.-C., ainsi que des traces circulaires occasionnées par le piétinement des ouvriers tirant les barres de l'appareil.

Les pressoirs ne sont donc attestés que sur quatre sites dont trois récemment signalés (fig. 61). Or, si les vestiges d'installations vinicoles d'Aquitaine n'ont pas toujours été correctement interprétés jusqu'ici, cela tient à cette absence presque totale de ruines de pressoirs. En Narbonnaise, les fouilles et les prospections conduites ces vingt dernières années ont permis en effet de retrouver plusieurs pressoirs en place et de nombreux blocs de pierre en provenant (150 environ pour le seul département du Var, $c f$. Brun, 1999, p. 155-158). Une part d'entre eux équipait des huileries, mais on sait qu'il n'y a pas de différence fondamentale entre pressoir à vin et à huile (Brun, 1993b); Caton les décrit en termes identiques. Même si, dans les régions d'intenses cultures et de forte érosion, les pressoirs, situés à un niveau plus élevé que les autres structures, ont été systématiquement détruits, il devrait subsister des blocs de pierre employés dans les fondations des pressoirs à levier : blocs d'assise de jumelles et de poteaux, contrepoids. Ils ne sont pas totalement inconnus dans l'Ouest puisqu'un bloc d'assise a été repéré à Saint-Plancard (Haute-Garonne) et un autre à Chênehutte-Trèves-Cunault en Maine-et-Loire (Provost, 1988a). Faute d'attention, une part de ces vestiges a pu échapper aux inventaires archéologiques, mais la disproportion est trop grande entre le nombre d'attestations de cuves à vin et celui des pressoirs.

On doit donc imaginer que les pressoirs étaient majoritairement d'un type différent de ceux bien connus en Narbonnaise. Le cas de Bapteste à Moncrabeau est éclairant : sans la minutieuse fouille de P. Jacques dans un secteur bien préservé car recouvert d'une mosaĩque, on n'aurait pas pu retrouver les vestiges fort ténus de l'emplacement du pressoir. Bien d'autres empreintes de pressoirs tout en bois et peut-être souvent à vis ont dû être détruites au cours des temps ou n'ont pas été repérées par les fouilles anciennes.

\section{LES CHAIS}

À l'exception de la villa de Bapteste à Moncrabeau, où il existait peut-être un chai abritant des dolia defossa et du site de Saint-Médard-de-Mussidan qui a livré deux dolia accolés, aucun bâtiment agricole d'Aquitaine n'a livré de ces alignements de jarres qui caractérisent les chais méditerranéens. Il ne semble donc pas que, à l'instar de la Lusitanie au I ${ }^{\text {er }}$ s. après J.-C. (Brun, 1997), la vinification ait été conduite dans des jarres : l'emploi de vaisseaux de bois se serait imposé d'emblée (fig. 83). Dans les installations les plus complètes (à La Haute-Sarrazine, Cognac et à La Pointe des Minimes, La Rochelle, à Soubise, à Allas-les-Mines, à Montgey, etc.), fouloirs et cuves sont associés à de longs bâtiments dont les sols, bétonnés ou non, présentent parfois des dispositions particulières permettant de les identifier. À La Haute-Sarrazine, le sol de la pièce 15 , recouvert de béton de tuileau, comportait deux rainures longitudinales semblant aboutir au bassin 5. À La Pointe des Minimes, les salles 3 et 13 présentaient aussi des sols bétonnés creusés de rigoles longitudinales aboutissant à des bassins. À Allas-les-Mines, la salle 1 et probablement les pièces 3 à 6 possédaient des sols en béton de tuileau en pente vers l'est, c'est-à-dire vers le fouloir et sa cuve, mais aucune rigole n'a été observée. Il faut rapprocher ces trois exemples de celui de La Moussigère à Puyréaux (fig. 84) : une vaste aire bétonnée est partagée longitudinalement en deux parties par une rigole qui relie deux cuves : l'une, rectangulaire et carrelée mesurant $1,60 \mathrm{~m}$ sur $1,20 \mathrm{~m}$ pour une profondeur de $0,80 \mathrm{~m}$; l'autre, de forme arrondie, enduite de béton de tuileau et mesurant $2,10 \mathrm{~m}$ sur 1,80 $\mathrm{m}$ (soit une contenance totale de $40 \mathrm{hl}$ ). Le sol de l'aire bétonnée est incliné vers la rigole de façon à canaliser un liquide vers les cuves. Cette curieuse installation n'a pas de parallèle exact. Ce pourrait ĉtre un fouloir à vin, mais, outre qu'il s'agirait d'un unicum, on ne voit pas comment on aurait pu retenir les raisins d'engorger la rigole dès le début du foulage. Il faut donc vraisemblablement rapprocher cet espace des pièces bétonnées de Cognac et de La Rochelle et l'interpréter comme un chai où étaient entreposés des foudres. Comme à La Pointe des Minimes et à La HauteSarrazine, la rigole centrale servait à recueillir le vin des 


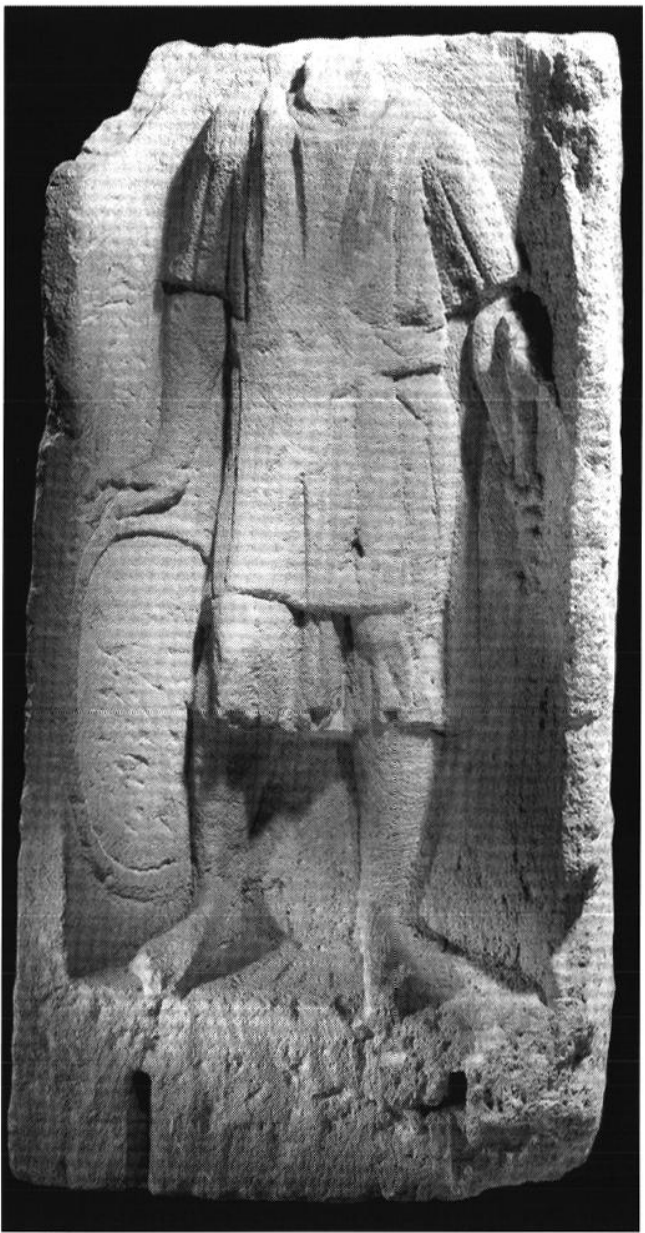

Fig. 83 - Monument funéraire d'un tonnelier, Bordeaux, Musée d'Aquitaine (cliché P. Bardou).

vaisseaux qui éclataient. Le dispositif est décrit par Palladius (Traité d'agriculture, I, 18) : "S'il est prévu de réserver un emplacement particulier aux foudres, il faut que cet emplacement soit établi sur des socles courts et [recouverts] d'un sol en mortier de tuileau comme celui du fouloir, afin que, si un foudre fuit sans qu'on s'en rende compte, les vins qui auront coulé, au lieu d'être perdus, soient recueillis dans une cuve placée en contrebas. » (traduction J.-P. Brun).

Les chais abritant les foudres sont de grandes salles longues de $9 \mathrm{~m}$ à plus de $60 \mathrm{~m}$ et larges de $5 \mathrm{~m}$ à $20 \mathrm{~m}$. Jusqu'à $10 \mathrm{~m}$ de largeur, il semble que les toitures aient été portées par une charpente à ferme, sans support intermédiaire, mais à Saint-Barthélemy de Montgey, le chai, qui mesure $20 \mathrm{~m}$ de largeur, est divisé en trois nefs par des piliers bien visibles sur la photographie aérienne (Passelac, 1986).
Stèle en calcaire anépigraphe qui était scellée par des crampons sur un soubassement. Incomplète en haut (elle a été sciée lors de son remploi). Éclats sur les arêtes, surtout à gauche.

Dimensions: $94 \mathrm{~cm} \times 48 \mathrm{~cm} \times 35 \mathrm{~cm}$.

Extraite du soubassement de la muraille gallo-romaine du Bas-Empire de Bordeaux, au centre de la face nord, en 1831.

Bordeaux, Musée d'Aquitaine, $n^{\circ}$ 60.2.80.

Datation : première moitié du III s. ? (d'après Braemer, 1959).

Dans une niche peu profonde, arrondie, voûtée en cul-de-four, se détache une représentation figurée en bas-relief: le personnage est debout, de face, vêtu d'une tunique s'arrêtant aux genoux, à manches courtes, couverte d'un tablier (de cuir ?) serré à la taille par une large ceinture. De sa main droite, il lève légèrement une aissette ou une herminette: un outil à manche court, à lame à l'extrémité large et horizontale, prolongée à l'arrière du manche par un marteau; en léger retrait, la main gauche est posée sur un bandeau circulaire représenté en perspective et allant jusqu'à la hauteur des chevilles. Le chiasme esquissé (bras droit plié et jambe gauche libre) suggère le mouvement. La stèle fait partie d'une importante série bordelaise de portraits en pied de défunts, où les scènes réalistes de personnages au travail, comme celle-ci, sont rares (Espérandieu, 1908, $n^{\text {os }} 1098,1099,1111$ ).

Dès la découverte, la scène a été interprétée comme un tonnelier au travail (Jouannet), ce qui dans la suite a été accepté avec (Espérandieu) ou sans (Braemer) hésitation. Mais Jullian n'a mentionné ce relief que comme celui d'un "jeune homme au cercle " et M. Reddé ne l'a pas retenu parmi les "scènes de métier" (Reddé, 1978). Pourtant, la tenue de l'homme, l'outil qu'il lève, le grand ovale de l'arrière plan qui semble être l'esquisse du dessus d'un tonneau donnent des raisons sérieuses de croire que l'on est bien en présence d'un tonnelier. BIBLIOGRAPHIE : Jouannet, 1832, p. 134 ; Jullian, 1890, p. 319 ; Espérandieu, 1908, $n^{\circ} 1112$; Braemer, 1959, $n^{\circ} 80$, p. 92 ; Bordeaux, 2000 ans d'histoire, 1970, p. 93, $n^{\circ} 72$ (L. Valensi).

Dans le Gers, ce type de long bâtiment, comportant généralement un espace aux sols maçonnés, a été repéré par les prospections aériennes de C. Petit-Aupert à Lectoure (Garros, Au Moulin et Cujon), à Marsolan (Garbeau), à Plieux (Bidot), à Castelnau-d'Arbieu (Gavachon) et peut-être à Pergain-Taillac (Saint-Martin) (Petit, 1997, fig. 26, 42, 46, 49, 51, 54, 64, 76). Les fouilles de Lestagnac (Saint-Mézard) ont renforcé l'hypothèse identifiant ces constructions comme des chais.

La lecture du tableau des dimensions des chais montre qu'à côté d'exploitations modestes certaines installations sont gigantesques, les plus grandes demeurant celles de Lestagnac (Saint-Mézard) dont l'ensemble vinicole mesure $80 \mathrm{~m}$ de longueur et celle de La HauteSarrazine (Cognac) qui devait compter au moins trois salles abritant des foudres et des tonneaux (tabl. IV) ; si chacune d'elles comportait deux files de vaisseaux, 
Tabl. IV - Dimensions des chais antiques d'Aquitaine.

\begin{tabular}{|lll|}
\hline \multicolumn{1}{|c|}{ Site } & \multicolumn{1}{c|}{ Longueur } & Largeur \\
\hline La Moussigère, Puyréaux & $9 \mathrm{~m}$ & $7,20 \mathrm{~m}$ \\
\hline Allas-les-Mines & $10,08 \mathrm{~m}$ & $5,32 \mathrm{~m}$ \\
\hline La Pointe des Minimes, La Rochelle : salle 3 & $15 \mathrm{~m}$ & $8 \mathrm{~m}$ \\
\hline La Haute-Sarrazine, Cognac : salle 15 & $16 \mathrm{~m}$ & $4,50 \mathrm{~m}$ \\
\hline La Haute-Sarrazine, Cognac : salle dans bât. B & $25 \mathrm{~m}$ environ & $8 \mathrm{~m}$ \\
\hline Péré Maillard, Soubise & $30 \mathrm{~m}$ au moins & $10 \mathrm{~m}$ \\
\hline Belmont, Royan & $28 \mathrm{~m}$ & $17 \mathrm{~m}$ \\
\hline Sainte-Colombe & $50 \mathrm{~m}$ & $14 \mathrm{~m}$ \\
\hline La Haute-Sarrazine, Cognac : salle dans bât. A & $66 \mathrm{~m}$ environ & $10 \mathrm{~m}$ \\
\hline Saint-Barthélemy, Montgey & $60 \mathrm{~m}$ au moins & $20 \mathrm{~m}$ \\
\hline Lestagnac, Saint-Mézard & $65 \mathrm{~m}(80 \mathrm{~m})$ & $15 \mathrm{~m}$ \\
\hline
\end{tabular}

l'exploitant disposait de plus de $200 \mathrm{~m}$ linéaires pour disposer ses fûts. Toutefois, à la différence des dolia dont on peut calculer précisément le nombre et la contenance, on ne peut fournir ici aucune estimation de production. Mais il faut dire que, pour La Haute-Sarrazine à Cognac, les capacités de vinification devaient approcher celles des plus grands domaines de Narbonnaise, tels que Le Molard à Donzère ou Les Toulons à Rians.

\section{INSTALLATIONS DE CHAUFFAGE}

Dans certaines villae, on trouve, à proximité des cuves, des vestiges de dispositifs de chauffage de deux types: chaudières et pièces sur hypocauste. Les deux équipe-

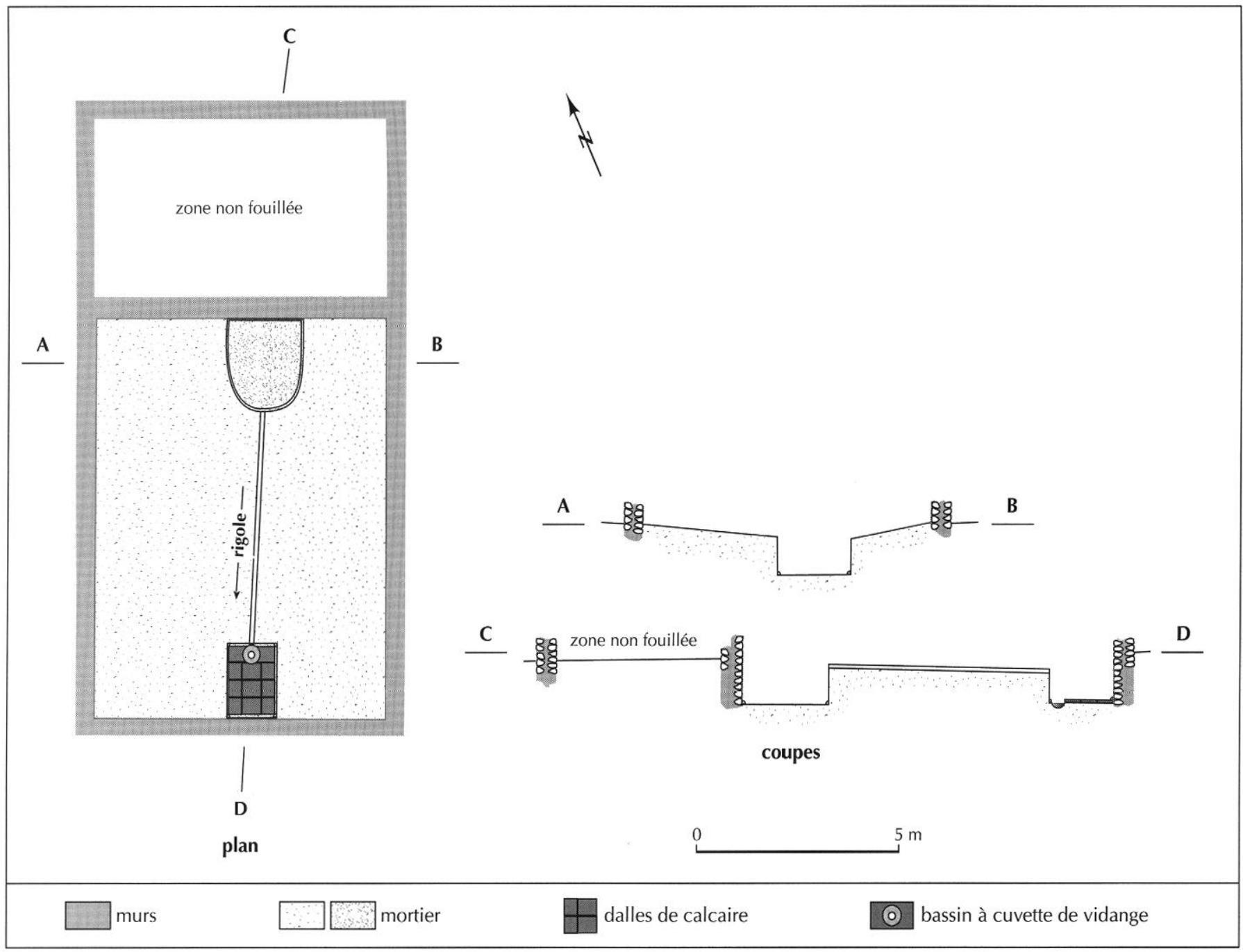

Fig. 84 - Plan et coupes de l'aire bélonnée à rigole centrale de La Moussigère à Puyréaux, Charente. Cette aire a pu servir de chai (dessin H. Gaillard, 1998, d'après un croquis de A. Favraud). 
ments sont certainement liés à la production du vin. Dans ces régions septentrionales par rapport à la Méditerranée, le raisin n'atteint pas une haute concentration en sucre, sauf exception comme le sauternes. En l'absence de traitement par le soufre, il convenait de renforcer la concentration des moûts si l'on voulait éviter que le vin ne se pique très rapidement. Une meilleure concentration en sucre et un plus haut degré alcoolique étaient obtenus en ajoutant des moûts concentrés (defrutum) au jus de foulage et de pressurage. Les recettes du defrutum et de la sapa sont bien connues grâce aux textes agronomiques antiques (Columelle, De l'agriculture, I, 6, 19). Dans certains cas, lors de vendanges pluvieues par exemple, on conseillait de faire chauffer tout le moût afin de provoquer l'évaporation d'une partie de l'eau (Palladius, Traité d'agriculture, XI, 14). Les chaudières sont donc fréquentes dans les chais ${ }^{180}$, mais elles ont été rarement observées, car, étant bâties en élévation, elles ont souvent disparu avec les sols. Le cas le plus net est celui de Belmont (Royan) (fig. 66 et 67 ), où le praefurnium de la salle sur hypocauste devait supporter une chaudière. Outre la réduction du moût, la chaleur était utilisée pour accélérer la maturation des vins. Les anciens recherchaient le goût de rancio, voire de madérisé, et tentaient de l'obtenir en chauffant les amphores soit au soleil ${ }^{181}$, soit dans des pièces situées au-dessus du foyer de la maison ${ }^{182}$, soit dans des apothèques construites en partie supérieure de foyers de thermes utilisés pour la maind'œuvre (Columelle, I, 6, 20), soit dans des salles sur hypocauste. Deux chais paraissent être également dotés de salles chaudes destinées à cette opération, ceux de Belmont à Royan et de Péré Maillard à Soubise. À Belmont, le praefurnium chauffe une pièce qui a $\mathrm{pu}$ avoir un double usage (apothèque et bain pour la maind'œuvre). À Péré Maillard, une pièce sur hypocauste voisine et semble-t-il communique avec une pièce au sol bétonné $\mathrm{B} 1$ qui pourrait correspondre à un fouloir.

L'installation de Belmont fonctionnait aux III $^{e}$ et IV ${ }^{\mathrm{e}}$ s. après J.-C., celle de Péré Maillard, paraît liée à la

180. Par exemple dans la villa de São Cucufate en Lusitanie.

181. Il n'y a pas d'installation vinicole en Égypte qui ne comporte son heliasterion, c'est-à-dire son enclos où l'on mettait les amphores à chauffer au soleil (P. Vindob. Sal., 12, 1.9 ; P. Strasb., VII, 696, 1, 8 ; P. Oxy., 729, 3638, 3491, 3354 ; P. Flor., I, 50 ; P. Ryl., II, 206 ; PSI, XIII, 328, 1. 43-44 ; P. Vat. Aphrod., 25 A, 1. 20).

182. C'est ainsi que procédait le père de Galien dans son domaine de Pergame (De antidotis, I, 3, voir J.-M. Jacques, 1996). réfection tardive du site au $\mathrm{IV}^{\mathrm{e}} \mathrm{s}$. À la même époque, plusieurs chais de la vallée de la Moselle sont équipés d'installations analogues ( $\mathrm{Im}$ Briesch à Piesport, Josefshof à Graach, Erden, voir infra, p. 168-174), alors qu'ils paraissent inconnus au Haut-Empire, non seulement en Aquitaine, mais aussi en Narbonnaise, où pourtant les fumaria de Marseille sont plusieurs fois décriés par Martial (voir supra, p. 70). Dans l'attente de données nouvelles, on peut émettre l'hypothèse que ce type particulier d'apothèque, construit sur hypocauste, ne s'est répandu en Gaule qu'au cours de l'Antiquité tardive.

J.-P. B.

\section{L'ÉVOLUTION DE LA VITICULTURE AQUITAINE DANS L'ANTIQUITÉ}

Les Bordelais rappellent volontiers les plus fameux témoignages de l'ancienneté de leur vin, ceux de Columelle et de Pline l'Ancien qui, à quelques années d'intervalle, citent la Biturica ${ }^{183}$, la vigne cultivée par les Bituriges Vivisques ${ }^{184}$, parmi les cépages les plus productifs de leur temps : ainsi, peu après le milieu du $\mathrm{I}^{\mathrm{er}} \mathrm{s}$. après J.-C., le vignoble de Bordeaux était déjà bien connu en Italie. Ces sources littéraires, qui constituent une donnée chronologique précieuse, sont aujourd'hui confirmées par la mise au jour de vestiges d'installations vinicoles dont les meilleurs exemples sont présentés ci-dessus. D'autres chantiers archéologiques ont, en outre, apporté un certain nombre d'indications complémentaires : grâce notamment aux fouilles urbaines, en particulier à celles de Bordeaux, les études carpologiques et amphoriques ont rapidement progressé; ce sont surtout celles-ci qui améliorent notre connaissance des origines de la viticulture aquitaine ${ }^{185}$. Aussi les débuts de

183. Columelle, De l'agriculture, III, 2, 19; Pline, $H$. N., XIV, 27-31 : Columelle écrivit son Agriculture probablement sous le règne de Néron et Pline l'Ancien publia son Histoire naturelle en 77 après J.-C. À propos de ces textes essentiels, on se reportera aux commentaires de Dion, 1959, p. 121-126, et d'Étienne, 1962, p. 102-109.

184. Les questions que soulèvent ces textes, en particulier l'attribution aux Bituriges Vivisques de cette Biturica, ont été examinées plus haut, p. 130.

185. En Aquitaine, depuis une dizaine d'années, les principales recherches sur les amphores ont été réalisées par F. Berthault qui nous a souvent fait profiter de son savoir; consulter principalement Berthault, 1988, 1991, 1997, 1998 ; Amiel, Berthault, 1996. 
la production du vin dans le sud-ouest de la Gaule commencent-ils à se préciser, et il est possible, dès à présent, d'esquisser un schéma de l'évolution de cette activité agricole.

\section{LA PREMIÈRE MOITIÉ DU I ${ }^{\text {er }}$ S. APRÈS J.-C. : LES DÉBUTS DE LA PRODUCTION DE VIN EN AQUITAINE}

On considère aujourd'hui que la culture de la vigne s'est diffusée précocement dans les pays de la Méditerranée occidentale, bien avant la conquête romaine, tant en péninsule Ibérique qu'en Gaule du SudEst ${ }^{186}$. Il est donc permis, malgré Strabon ${ }^{187}$, de se poser la même question pour l'Aquitaine, au moins pour ses contrées les plus méridionales. Même s'ils sont encore fort rares et sujets à controverse, quelques indices paraissent signifier que la culture de la vigne a été pratiquée en Gaule non méditerranéenne avant la période romaine ${ }^{188}$ : il s'agit de pépins de raisin de vigne cultivée (Vitis vinifera) et d'une serpette découverts à Agen (Marinval, 1994, p. 47-49), ainsi que de sarments taillés recueillis à Lectoure (Larrieu-Duler, 1973, p. 43), trouvailles effectuées dans des puits à offrandes datés de la fin du second âge du Fer, entre la fin du $\mathrm{II}^{\mathrm{c}} \mathrm{s}$. avant J.-C. et l'époque augustéenne ${ }^{189}$.

Ces découvertes, qui très probablement indiquent une culture de la vigne au $\mathrm{I}^{\mathrm{er}}$ s. avant J.-C., laissent aussi supposer une vinification, mais n'en constituent pas une preuve incontestable. Aussi, pour le moment, aucune production de vin n'est parfaitement attestée en Aquitaine avant le début de notre ère. En fait, pour l'ordre d'apparition des vignobles, on en reste encore à

186. Des vestiges d'installations pour la production de vin y ont même été mis au jour ; à propos de cette viticulture préromaine, voir en particulier pour la péninsule Ibérique: Gómez Bellard et al., 1993b, et pour la Gaule, supra, p. 71-72.

187. Géographie, II, 1, 16. Strabon indique que la vigne ne pousse pas en Celtique océanique. L'information, probablement tirée de Poseidonios, est sans doute valable pour les années 100 avant J.C. environ, mais elle était peut-être erronće à l'ćpoque augustéenne, quand le géographe écrivait.

188. Comme le pense Marinval, 1994, p. 50. Il est toutefois possible que les pépins aient été apportés avec les amphores italiques; sur cette question voir suprap. 72.

189. Pour le puits d'Agen, voir Boudet dir., 1994, p. 86-88; pour celui de Lectoure, voir Larrieu-Duler, 1973, p. 11-67. celui que proposa $R$. Dion qui tenait pour la priorité du vin de Gaillac (Dion, 1959, p. 105-107). Cette hypothèse d'une production de vin en Gaillacois dès l'époque augustéenne, qui paraissait assez. mal étayée lorsqu'elle fut émise il y a quarante ans, est maintenant assurée grâce aux trouvailles effectuées à Montans par T. Martin : dans ce grand centre de production de céramique sigillée, situé au bord du Tarn, en face de Gaillac, on fabriquait des amphores dès le premier quart du $\mathrm{I}^{\mathrm{er}} \mathrm{s}$. après J.-C., en particulier une amphore vinaire de forme Pascual $1^{190}$; un vin était donc produit dans les campagnes voisines dès cette époque et il était exporté dans ce récipient par les bateaux du Tarn et de la Garonne ${ }^{191}$.

Vraisemblablement d'autres vignobles se sont constitués pendant cette première moitié du $\mathrm{I}^{\mathrm{er}} \mathrm{s}$., mais on ne dispose pas encore de témoignage aussi sûr que pour le Gaillacois. On signalera cependant la présence de pépins de raisin dans une couche peut-être augustéenne à Bergerac (Gaillard, 1997, p. 72) et, surtout, la découverte de ceps de vigne dans un niveau du début du $\mathrm{I}^{\mathrm{er}}$ s. à Bordeaux ${ }^{192}$.

Toutefois, comme pour Montans, ce sont seulement les études sur les amphores qui permettent aujourd'hui de préciser la chronologie de la viticulture en Aquitaine et de situer approximativement sous le règne de Claude le début de l'exportation de vin de plusieurs vignobles, en particulier ceux de Bordeaux, d'Agen et de Périgueux. Grâce à des analyses de pâtes, F. Berthault a montré qu'on a produit dans ces villes, ou à proximité, des amphores dont les formes sont identiques à celles qui servaient au transport des vins des grands vignobles méditerranéens de la première moitié du $I^{\mathrm{er}} \mathrm{s}$. : c'est assurément pour exporter un vin produit déjà en quantités importantes que les Petrucores ont imité, vers 50 après J.-C., les amphores de formes Dressel 2/4 et Gauloise 3/5 (Berthault, 1998, p. 452 et fig. 3, $\mathrm{n}^{\circ}$ 5), les Nitiobroges, vers 50-60, le type Oberaden 74 (Berthault, 1998, p. 452

190. De nombreux ratés de cuisson ont été recucillis et un exemplaire porte la marque Tarani, à rapprocher sans doute du nom du fleuve qui baignait ce vignoble (Martin, 1996, p. 44-45 et fig. 54).

191. Le Tarn était navigable depuis Albi : les embarcations de Philon, l'intendant d'Ausone devenu commerçant, l'empruntaient. (Ausone, Epist., XXII, 31-32) ; sur ces embarcations, voir Étienne, 1972 et Martin, 1996 , p. $50-51$.

192. Fouille du quartier Saint-Christoly: voir Debord, Gauthier, Rapport de fouilles cité supra note 24, p. 95, et fig. 67, p. 105 : dans ce niveau (US 1763) ont également été reconnus des trous de poteaux des premières cabanes construites sur la rive gauche de la Devèze. Voir aussi supra, p. 150-151. 
et fig. $3, \mathrm{n}^{\circ} 3$ ) et les Bituriges Vivisques le type Dressel 28, entre 50 et $80^{193}$.

\section{LA SECONDE MOITIÉ DU I ${ }^{\text {er }}$ S. : L'ESSOR DE LA VITICULTURE AQUITAINE}

Quelques décennies plus tard, la viticulture aquitaine a connu un développement rapide, favorisé sans doute par l'ouverture du marché breton (Étienne, 1962, p. 102). Cet essor s'est manifesté par l'apparition de nouveaux vignobles, qui ont aussi été révélés par l'identification d'imitations locales d'amphores : à Saintes, une production d'amphores de formes Dressel 2/4 et Gauloise 3/5 est attestée sous les Flaviens (Berthault, 1991) ; au Langon (Vendée), c'est le type Dressel 2/4 qui fut repris à la fin du $\mathrm{I}^{\mathrm{er}}$ s. (Berthault, 1997).

Mais $F$. Berthault a, en outre, découvert que cette croissance de la production viticole dans le sud-ouest de la Gaule s'est accompagnée de la création d'un type d'amphore nouveau qu'avec raison il désigne par le nom d'amphore d'Aquitaine. Ce récipient spécifique, destiné à diffuser le vin d'Aquitaine en le distinguant des autres crus, apparut vers 70 après J.-C. ${ }^{194}$. Ainsi, grâce au matériel amphorique, il est certain aujourd'hui que la grande production des vignobles de l'Aquitaine a commencé sous le règne de Vespasien. N'oublions pas que c'est à cette même époque que la réputation de la Biturica avait atteint Rome (voir supra, p. 130).

Observe-t-on en même temps la construction de nombreuses installations de vinification? Il est difficile de l'affirmer pour le moment, d'après les vestiges. Rappelons que les chronologies disponibles sont presque toujours imprécises, sinon sujettes à caution ${ }^{195}$, et, surtout, que les chais eux-mêmes sont très rarement datés de façon convenable. Ainsi, les bassins de FontBelle à Segonzac (Vernou et al., 1993, p. 208), de PetitMoulin à Baignes (Vernou et al., 1993, p. 59), de Reignac (Robin, 1995 et supra, p. 141-142), des Murasses à Lugasson (Petit, 1988) et des Moutiers à Rochefort

193. Amphores de la place Camille-Jullian, d'après Berthault, 1998, p. 452 et fig. $2, n^{\circ} 3$.

194. Datation établie grâce aux fouilles récentes de Bordeaux, spécialement celles du puits de la place de la République (Berthault, 1988).

195. Comme on a été obligé de le constater plusieurs fois supra, p. 134, 144,146 et 148 .
(Maurin, 1999, p. 231) appartiennent certes à des villae qui furent édifiées au cours du $\mathrm{I}^{\mathrm{er}} \mathrm{s}$., mais nous ne disposons d'aucune chronologie pour les bassins euxmêmes.

En fait, les quelques données disponibles semblent indiquer un léger décalage chronologique entre cet essor des vins d'Aquitaine et l'équipement en dur des chais des villae. Les seules datations apparemment satisfaisantes ne concernent que quelques sites : il s'agit des bâtiments de vinification des Chapelles à Port-des-Barques et de La Pointe des Minimes à La Rochelle qui ont vraisemblablement été construits à la fin du I Irr $\mathrm{s}$. et de l'aile vinicole de Bapteste qui a été ajoutée à la villa au début du $\mathrm{II}^{\mathrm{e}} \mathrm{s}$. Quant à la datation proposée pour le premier état de l'installation de La Haute-Sarrazine, sa marge d'incertitude est grande, mais il est vraisemblable que cet établissement était en service durant le $\mathrm{II}^{\mathrm{e}} \mathrm{s}$.

Il semble donc, pour l'instant, que les premiers équipements de vinification n'aient guère laissé de traces ; du moins, n'ont-ils pas encore été reconnus. Est-ce parce que la plupart étaient en bois, même les cuves ? Ensuite, vers 100 après J.-C., ils auraient été remplacés par des ensembles plus vastes, dotés de fouloirs, de pressoirs et de bassins construits en dur.

\section{UNE DURÉE APPROXIMATIVE DE DEUX SIÈCLES}

Ces équipements vinicoles furent ensuite parfois améliorés et agrandis, notamment ceux de La HauteSarrazine et de La Pointe des Minimes, ainsi que celui d'Allas-les-Mines qui a été transformé (voir supra, p. 136, 144, 147 et 148). D'autres établissements viticoles sont aussi apparus au cours du Haut-Empire, comme par exemple celui de Belmont.

L'apogée du vignoble aquitain semble se situer au II $^{e} s$. La durée de cette première viticulture est d'ailleurs assez bien fixée, car on a constaté que de nombreux établissements présentent la même évolution chronologique : la plupart restèrent en activité pendant tout le $\mathrm{II}^{\mathrm{e}} \mathbf{s} .{ }^{196}$ et disparurent à peu près en même temps, au cours de la seconde moitié du $\mathrm{III}^{\mathrm{e}} \mathrm{s}$. (cf. chronologies, supra, p. 136, 144 et 148). La datation des abandons est

196. À quelques exceptions près, notamment celle de l'établissement de Font-Belle à Segonzac, qui fut, semble-t-il, beaucoup plus éphémère (Vernou et al., 1991). 
souvent bien établie, en raison de la quantité de céramiques jetées dans les bassins pour les combler: la plupart des installations connues, en particulier celles de Ia Haute-Sarrazine (voir supra, p. 142-144), d'Allasles-Mines (voir supra, p. 136), de La Pointe des Minimes (Flouret et al., 1984, p. 17), ainsi, sans doute, que celles du Haut-Pampin (Maurin, 1999, p. 181-182) et de Cadillac (Coupry, 1957), ont cessé de fonctionner après 250.

Ainsi, la grande production des vins d'Aquitaine a duré à peu près deux siècles, de 50-70 à 250-270 environ. Ajoutons que, si les débuts de cette viticulture ont seulement été datés grâce aux études sur les amphores locales, son déclin ne peut être reconnu qu'en se fondant sur la destruction des établissements viticoles, puisque les amphores n'étaient plus guère employées à cette époque: elles avaient été délaissées environ un siècle plus tôt, vraisemblablement au profit du tonneau.

\section{FIN III' ${ }^{\text {S.-VI }}{ }^{\mathrm{e}}$ S. : CRISE, RESTAURATION ET MUTATIONS}

L'abandon de nombreuses installations vinicoles pendant la seconde moitié du III' ${ }^{\mathrm{e}} \mathrm{s}$. semble indiquer une crise agricole et des mutations. Toutefois la viticulture aquitaine s'est maintenuc puisqu'elle est assez fréquemment attestée au IV es.

Les textes d'Ausone montrent l'importance de cette culture dans le fundus des villae du poète bordelais, et en particulier dans sa célèbre villula (Ausone, De herediolo, XII, 2, 9 et 21-24) : ce " petit héritage » comportait 25 ha de vignes, soit environ un dizième de la superficie du fundus et, surtout, un tiers de ses surfaces cultivées, ce qui représente un important vignoble et laisse supposer une considérable production de vin, de l'ordre d'un millier d'hectolitres par an ${ }^{197}$.

Les découvertes archéologiques confirment ces sources littéraires et attestent la production de vin en Aquitaine au cours de l'Antiquité tardive. Elles montrent la survivance, souvent avec des transformations, des équipements anciens. En effet, quelques établissements du Haut-Empire ont continué à être utilisés après le $\mathrm{III}^{\mathrm{e}} \mathrm{s}$. : c'est assurément le cas des installations de Belmont et des

197. En reprenant les donnćes sur les rendements établies pour les vignes d'Italie par Tchernia, 1995b.
Chapelles à Port-des-Barques (voir supra, p. 140-141). Pour d'autres établissements fouillés anciennement, les informations sont davantage sujettes à caution; il n'est pas impossible cependant que l'installation de Nodin à Montcaret (Gaillard, 1997, p. 165) et celle de SainteColombe (Coste, 1969 et supra, p. 148-149) aient fonctionné à la fin de l'Antiquité ; on pourrait se poser aussi la question pour les bassins de Mérignas et de Soubise (Duprat, 1986; Papinot, 1989; Maurin, 1999, p. 294-295). Signalons enfin la présence de pollens de vigne à Bordeaux dans des niveaux du $\mathrm{IV}^{\mathrm{e}}-\mathrm{V}^{\mathrm{e}} \mathrm{s}$. (voir supra, p. 151).

Que devint ce vignoble pendant le haut Moyen Âge ? Il faut constater que les vestiges d'équipements de vinification font presque totalement défaut à partir du $\mathrm{V}^{\mathrm{e}} \mathrm{s} .{ }^{198}$. On s'est rendu compte aussi que quelques vins méditerranéens parvenaient dans le sud-ouest de la France pendant l'Antiquité tardive ${ }^{199}$. Toutefois, il est certain que la viticulture a subsisté en Aquitaine car elle est bien attestée par un certain nombre de textes: comme au temps d'Ausone, les domaines des environs de Bordeaux comprenaient toujours des vignes au $\mathrm{VI}^{e}$ et $\mathrm{VII}^{e}$ s., en particulier à Plassac, Floirac et Preignac ${ }^{200}$; plus à l'est, des vignobles existaient aussi au VII $\mathbf{s} .{ }^{201}$. Il est même vraisemblable que la culture de la vigne se soit diffusée dès cette époque sur des terroirs beaucoup moins favorables, par exemple dans le Massif central, en raison, notamment, de l'importance liturgique du vin et du déclin des relations commerciales ${ }^{202}$.

C. P.A. et P. S.

198. À l'exception peut-être des bassins de Nodin près de Montcaret, qui auraient fonctionné encore au $\mathrm{V}^{\mathrm{e}} \mathrm{s}$.

199. Mais il ne peut s'agir que de très faibles quantités de vins de prestige destinés aux élites. À propos de ces importations reconnues grâce à la présence d'amphores orientales, notamment de Gaza, dans le Sud-Ouest, voir Amiel, Berthault, 1996. Ce vin de Gaza est encore mentionné par Grégoire de Tours au VI' s. (Hist. Franc., VII, 29).

200. Villae de Plassac et de Floirac connues par le testament de Bertechramnus, évêque du Mans, qui fut rédigé en 615-616 (éd. Pardessus, Diplomala, I, p. 206-207, en dernier lieu Weidemann, 1986), vignoble à Preignac de Léonce le Jeune, évêque de Bordeaux au milieu du $\mathrm{Vl}^{\mathrm{e}} \mathrm{s}$. (Venance Fortunat, Poèmes, I, 20). À propos de ce vignoble de l'Antiquité tardive, voir Higounet, 1963, p. 223-224.

201. En particulier dans les villae de Nizezius: Charte de Nizezius, rédigée en 680 à Moissac (éd. C. Devic et J. Vaissette, Histoire générale du Languedoc, $2^{\mathrm{e}}$ ćd., 1875, II, col. 42-45).

202. La communion sous les deux espèces fut accordée aux tidèles jusqu'au XII"s. 